TABLES FOR DERIVING DIFFUSION COEFFICIENTS FROM CONCENTRATION GRADIENTS

IN CYLINDRICAL RODS
A. H. Dexter
SRL $.01 \%$
W. V. Baxter
RECORD COPY

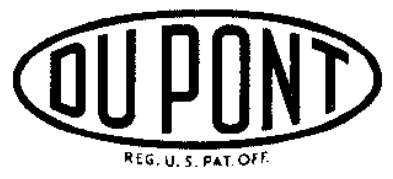

Savannah River Laboratory

Aiken, South Carolina 


\section{LEGAL NOTICE}

This report was prepered as an account of Government sponsored work. Nelther tho Unsted Btaten, nor the Commisesion, nor any person acting on behalf of the Comensoston:

A. Makes eny warranty or rapresentation, expressed or implied, with respect to the sccuracy. completeness, or ussfildeess of the informaton contalned in this report, or that the we of any information, spparatus, method, or proceses discioned in thes report may not iniringe privately ouned righto; or

B. Acsumes any llabsititien with respect to the use of, or for damagen reacultung from the of ayy tnformation, apparatus, method, or proceso disclosed in this report.

As uged in the above. "person Reting on behalf of the Commlasion" includes any etoployee or contractor of the commiswion, or employee of such contrector, to the extent that such employee or contractor of the Commisaton, or employee of auch contrictor prepares.

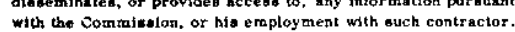

Printed in the United States of America Available from

Clearinghouse for Federal Scientific and Technical Information National Bureau of Standards, U. S. Department of Commerce Springfield, Virginia 22151

Price: Printed Copy $\$ 3.00 ;$ Microfiche $\$ 0.65$ 
DP -1111

Meta1s, Ceramics, and Materials

(TID-4500)

\title{
TABLES FOR DERIVING DIFFUSION COEFFICIENTS FROM CONCENTRATION GRADIENTS IN CYLINDRICAL RODS
}

\author{
by \\ Arthur H. Dexter \\ Nuclear Materials Division \\ and \\ William V. Baxter \\ Applied Mathematics Division \\ Approved by \\ P. H. Permar, Research Manager \\ Nuclear Materials Division
}

November 1967

E. I. DU PONT DE NEMOURS \& COMPANY

SAVANNAH RIVER LABORATORY

AIKEN, S. C. 29801

CONTRACT AT(07.2)-1 WITH THE

UNITED STATES ATOMIC ENERGY COMMISSION 


\begin{abstract}
For problems involving diffusion in cylindrical rods, computer-calculated tables are provided from which the experimentalist can readily calculate the diffusion coefficient $D$ from the measured concentration gradient in the rod.
\end{abstract}




\section{INTRODUCTION}

The diffusion coefficient of a solute in a metal may be determined by immersing a cylindrical rod of the metal in the solute and measuring the concentration gradient of the solute in the rod after a known exposure time.

Cylindrical rods are convenient specimens for diffusion studies because:

- The solute concentration gradient is symmetrical with respect to the axis of the rod.

- Rods can be machined or chemically milled in increments so the solute concentration can be determined as a function of the radius.

This report contains tables that facilitate the calculation of diffusion coefficients from concentration gradients measured in cylindrical rods. The tables were calculated from a solution of the cylindrical diffusion equation on an IBM System/360-65.

The tables are entered with dimensionless numbers $C / C_{0}$ and $r / a$, where $C$ is the gas concentration at radius $r$ in a cylindrical rod, with an outer radius $a$ and a gas concentration $c_{0}$ at $a$. A third dimensionless number $D t / a^{2}$, which corresponds to the $C / C_{0}$ and $r / a$ values, is read out from the tables. A simple substitution of $a^{2}$ and the exposure time $t$ of the rod to the gas provides the diffusion coefficient $D$. The tables include values of :

- $x / a$ from 0.20 to 1.0 in increments of 0.01

- $D t / a^{2}$ from 0.001 to 0.1 in increments of 0.001

- $C / C_{0}$ from approximately 0.1 to $10^{-5}$ 
These tables supplement a published graphical representation, ${ }^{1}, 2$ which is restrictive in values of $D t / a^{2}$, requires interpolation, and is unusable for the small values of $C / C_{0}\left(10^{-3}\right.$ to $\left.10^{-5}\right)$. The computer-calculated information is presented in tabular form rather than in graphic, because a graph of publishable size would be too difficult to read accurately.

\section{EQUATIONS}

The tables were generated by evaluating the formula ${ }^{2}$ :

$$
\frac{C}{C_{0}}=1-2 \sum_{n=1}^{\infty} \frac{e^{-\beta_{n}^{2} D t / a^{2}} J_{o}\left(\beta_{n} r / a\right)}{\beta_{n} J_{1}\left(\beta_{n}\right)}
$$

The above is a solution of the diffusion equation relative to a cylinder and a radial direction,

$$
\frac{\mathrm{d}^{2} \mathrm{C}}{\mathrm{dr}}+\frac{1}{\mathrm{r}} \frac{\mathrm{dC}}{\mathrm{dr}}+\alpha_{\mathrm{n}}^{2} \mathrm{C}=0
$$

as applied to a cylinder of radius $a$, having a constant surface concentration $C_{0}$ and initial internal concentrations of zero.

Additional initial conditions of

$$
C=C_{0}, r=a, t \geqslant 0
$$

were used to obtain (1) from the general solution of (2),

$$
C=\sum_{n=1}^{\infty} A_{n} J_{o}\left(\alpha_{n} r\right) e^{-D t} \alpha_{n}^{2}
$$

J. Crank. The Mathematics of Diffusion. Oxford, Clarendon Press (1964), pages 64-67.

2 H. S. Carslaw and J. C. Jaeger. Conduction of Heat in Solids. Oxford, Clarendon Press (1950), page 201. 
The values $\beta_{n}=a \alpha_{n}$ are the positive roots of

$$
J_{0}\left(a \alpha_{n}\right)=0
$$

Alternative solutions of the cylindrical diffusion equation are available, but these were not used because of limitations on values of $r / a$ and exposure time $t$. One such solution (1), which involves the complementary error function, is

$$
\begin{gathered}
\frac{C}{C_{0}}=\frac{a^{1 / 2}}{r^{1 / 2}} \operatorname{erfc} \frac{a-r}{2(D t)^{1 / 2}}+\frac{(a-r)(D t a)^{1 / 2}}{4 a r^{3 / 2}} i \operatorname{erfc} \frac{a-r}{2(D t)^{1 / 2}}+ \\
\frac{\left(9 a^{2}-2 a r-7 r^{2}\right)}{32 a^{3 / 2} r^{5 / 2}} i^{2} \operatorname{erfc} \frac{a-r}{2(D t)^{1 / 2}}+\cdots
\end{gathered}
$$

This could be used for small values of $t$ where $r / a$ is not small.

\section{DETAILS OF THE CALCULATION}

In order to assure accuracy of the tables over the desired range, the double-precision version of $\mathrm{BESJ}^{3}$ was employed. Successive terms in each summation were evaluated until a term was found whose magnitude differed from that of the sum by a factor of $10^{-10}$. A maximum of 45 terms was required in the formulation of $C / C_{0}$, corresponding to the combination of the lowest values of $D t / a^{2}$ and $r / a$.

All of the available (150) published values ${ }^{4}$ for the Bessel roots $\beta_{n}$ and the functions $J_{1}\left(\beta_{n}\right)$ were supplied to the program. Since a maximum of 45 terms was used, only the first 45 values were necessary. A minimum of only 5 terms was required for higher values of $D t / a^{2}$ and $x / a$; e.g., 0.1 and 0.75 , respectively.

3 IBM Corporation. "System/360 Scientific Subroutine Package (360A-CM-03X), Version II." Programmer's Manual H20-0205-1 (1967), page 156 .

4 British Association for the Advancement of Science. Mathematical Tables, Volume VI, Bessel Functions, Part I. University Press (1950). 


\section{CONCLUSIONS}

The tables provided on the next 10 pages are arranged so that the user enters the tables with a given value of $r / a$, scans across until he reaches the measured value of $C / C_{0}$ corresponding to the $r / a$, and then reads the $D t / \alpha^{2}$ value from the abscissa at the top of the page. Substitution of $a^{2}$ and the exposure time $t$ of the rod to the solute yields the diffusion coefficient $D$. 
TABLES OF CONCENTRATION RATIOS - c/Co

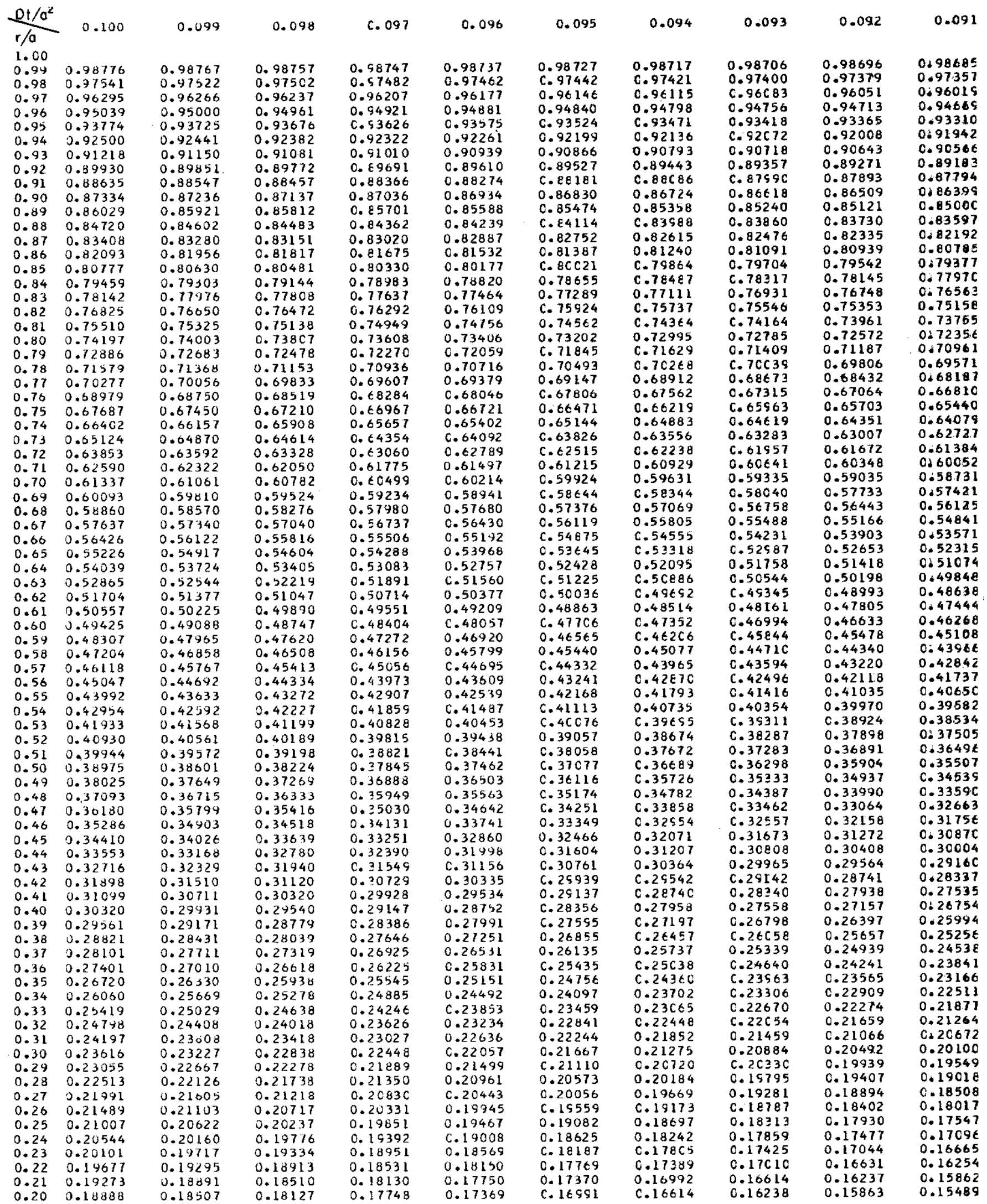


TABLES OF CONCENTRATION RATIOS - $C / C_{0}$

\begin{tabular}{|c|c|c|c|c|c|c|c|c|c|c|}
\hline$\frac{0+/ a^{2}}{r / a}$ & 0.090 & 0.089 & 0.088 & 0.087 & 0.086 & 0.085 & C.C \&4 & $0 . c 83$ & 0.082 & 0.081 \\
\hline $\begin{array}{l}1.00 \\
0.99\end{array}$ & 0.98674 & 0.98663 & 0.98651 & 0.98640 & 0.98628 & C. $S E \in 16$ & C. $9 \& \in C 4$ & C. 98592 & & \\
\hline 0.58 & 0.97335 & 0.97313 & 0.97290 & 0.97267 & $\begin{array}{l}0.97244 \\
0.95848\end{array}$ & 0.97220 & 0.97196 & 0.97171 & $\begin{array}{l}C .97146 \\
0.95700\end{array}$ & $\begin{array}{l}0.97121 \\
0.95662\end{array}$ \\
\hline 0.97 & 0.95985 & 0.95952 & $\begin{array}{l}0.95918 \\
0.94534\end{array}$ & $\begin{array}{l}0.95883 \\
0.94488\end{array}$ & $\begin{array}{l}0.95848 \\
0.94440\end{array}$ & $\begin{array}{l}\text { C. } 95812 \\
\text { C. } 94353\end{array}$ & $\begin{array}{l}0.95775 \\
c .94344\end{array}$ & $\begin{array}{l}0.95738 \\
0.94294\end{array}$ & $\begin{array}{l}0.951700 \\
0.94244\end{array}$ & $\begin{array}{l}0.95662 \\
0.94192\end{array}$ \\
\hline $\begin{array}{l}0.96 \\
0.95\end{array}$ & $\begin{array}{l}0.94625 \\
0.93254\end{array}$ & $\begin{array}{l}0.94580 \\
0.93198\end{array}$ & $\begin{array}{l}0.94534 \\
0.93141\end{array}$ & $\begin{array}{l}0.94488 \\
0.93083\end{array}$ & $\begin{array}{l}0.94440 \\
0.93023\end{array}$ & $\begin{array}{l}c .34333 \\
0.92963\end{array}$ & 0.92902 & 0.92840 & 0.92777 & 0.92712 \\
\hline 0.94 & 0.91875 & 0.91807 & 0.91738 & 0.51668 & c. 91597 & C. 91525 & 0.91451 & 0.91377 & 0.91301 & 0.91223 \\
\hline 0.93 & 0.70488 & 0.90409 & 0.90328 & 0.90246 & 0.90163 & C. $\operatorname{scc} 79$ & C.89993 & 0.89905 & 0.89816 & 0.89736 \\
\hline 0.92 & 0.89094 & 0.89003 & 0.88911 & 0.88817 & 0.88722 & 0.88625 & C.88527 & C.88427 & 0.88325 & 0.88222 \\
\hline 0.91 & 0.87693 & 0.87591 & 0.87487 & 0.87382 & 0.87275 & 0.87166 & 0.87055 & 0.86942 & 0.86828 & 0.86712 \\
\hline 0.90 & $\begin{array}{l}0.46287 \\
0.84877\end{array}$ & $\begin{array}{l}0.86174 \\
0.84752\end{array}$ & 0.86058 & 0.85941 & $\begin{array}{l}0.85822 \\
0.84366\end{array}$ & $\begin{array}{l}\text { C. } 85701 \\
0.84232\end{array}$ & $\begin{array}{l}C .85578 \\
0.84 C 57\end{array}$ & $\begin{array}{l}0.85453 \\
C .83560\end{array}$ & $\begin{array}{l}0.85326 \\
0.83820\end{array}$ & $\begin{array}{l}0.85197 \\
0.83678\end{array}$ \\
\hline $\begin{array}{l}0.89 \\
0.88\end{array}$ & & $\begin{array}{l}0.84752 \\
0.83327\end{array}$ & $\begin{array}{l}0.84625 \\
0.83189\end{array}$ & $\begin{array}{l}0.84496 \\
0.83048\end{array}$ & $\begin{array}{l}0.84366 \\
0.82906\end{array}$ & 0.82761 & 0.82613 & 0.82463 & 0.82311 & 0.82156 \\
\hline 0.87 & 0.32047 & 0.81900 & 0.81750 & C.81598 & 0.81444 & C. 81287 & 0.81127 & 0.80965 & 0.80800 & $0+80633$ \\
\hline 0.86 & 0.80629 & 0.80471 & 0.80310 & 0.80146 & 0.79980 & 0.79812 & $0.7964 \mathrm{C}$ & c. 79460 & 0.79289 & 0.79109 \\
\hline 0.85 & 0.79211 & 0.79041 & 0.78869 & 0.78694 & 0.78517 & 0.78337 & 0.78153 & $c .77967$ & 0.77178 & $0.7758 t$ \\
\hline 0.84 & 0.77792 & 0.77612 & 0.77429 & 0.77243 & C. 77054 & 0.76862 & 0.76667 & 0.76469 & 0.76268 & 0.76064 \\
\hline 0.83 & 0.76374 & 0.76183 & 0.75989 & 0.75793 & 0.75593 & 0.75390 & 0.75183 & C. 74974 & 0.74761 & 0.74544 \\
\hline 0.82 & 0.74959 & 0.74157 & 0.74553 & 0.74345 & 0.74134 & 0.73920 & 0.73702 & 0.73481 & 0.73257 & $\begin{array}{l}0.73028 \\
0.71517\end{array}$ \\
\hline 0.81 & 0.73546 & 0.73334 & 0.73119 & 0.72900 & $\begin{array}{l}0.72679 \\
0.71228\end{array}$ & $\begin{array}{l}0.72454 \\
\text { c.7cs52 }\end{array}$ & & 0.71993 & 0.71757 & \\
\hline $\begin{array}{l}0.8 .0 \\
0.79\end{array}$ & $\begin{array}{l}0.72137 \\
0.70732\end{array}$ & $\begin{array}{l}0.71914 \\
0.70500\end{array}$ & $\begin{array}{l}0.71689 \\
0.70264\end{array}$ & $\begin{array}{l}0.71460 \\
0.70025\end{array}$ & $\begin{array}{l}0.71228 \\
0.69782\end{array}$ & $\begin{array}{l}C .76952 \\
0.69536\end{array}$ & $\begin{array}{l}C .7 C 753 \\
0.69286\end{array}$ & $\begin{array}{l}0.7 C 509 \\
0.69032\end{array}$ & $\begin{array}{l}0.70262 \\
0.68774\end{array}$ & $\begin{array}{l}0.70011 \\
0.68512\end{array}$ \\
\hline 0.78 & 0.64332 & 0.69090 & 0.68845 & c. 68596 & 0.68343 & 0.68086 & 0.67826 & 0.67561 & 0.67293 & $0.6702 \mathrm{C}$ \\
\hline 0.77 & 0.67939 & 0.67687 & 0.67432 & 0.67173 & 0.66911 & C. $6 \in \in 44$ & 0.66374 & 0.66099 & 0.65820 & 0.65537 \\
\hline 0.76 & 0.66553 & 0.66292 & 0.66027 & 0.65759 & 0.65486 & $0 . t 5210$ & C.64930 & $0.64 t 45$ & C. 64356 & 0.64063 \\
\hline 0.75 & 0.65174 & 0.64904 & 0.64630 & 0.64352 & 0.64071 & 0.63785 & 0.63490 & 0.63201 & 0.62902 & 0.62595 \\
\hline 0.74 & 0.63804 & 0.63525 & 0.63242 & 0.62955 & 0.62664 & C. $6237 \mathrm{C}$ & $c .62670$ & 0.61767 & 0.61459 & 0.61146 \\
\hline 0.73 & 0.62443 & 0.62155 & 0.61864 & 0.61568 & 0.61269 & $c .609 t 5$ & $c .6 c \in 57$ & $c .60344$ & 0.60027 & 0.59705 \\
\hline 0.72 & 0.61092 & 0.60796 & 0.60496 & 0.60192 & 0.57884 & 0.59572 & 0.59255 & 0.58934 & 0.58608 & 0.58277 \\
\hline 0.71 & 0.59752 & 0.59448 & 0.59140 & 0.58828 & 0.58511 & c. 58191 & 0.57866 & 0.57536 & 0.57202 & 0.56863 \\
\hline 0.70 & 0.58423 & 0.58111 & 0.57795 & 0.57475 & 0.57151 & 0.56823 & C. $5 \in 4 S C$ & C. $5 \in 152$ & 0.55810 & 0.55463 \\
\hline 0.69 & 0.57106 & 0.56787 & 0.56464 & 0.56136 & 0.55804 & 0.55468 & 0.55128 & 0.54782 & 0.54432 & $0.5407 E$ \\
\hline 0.68 & 0.55802 & 0.55476 & 0.55145 & $0.5481 \mathrm{C}$ & C. 54471 & C. 54128 & C. 53780 & 0.53428 & 0.53070 & 0.52708 \\
\hline 0.67 & 0.54511 & 0.54178 & 0.53841 & 0.53499 & 0.53153 & 0.52803 & C. 52448 & C. 52089 & 0.51724 & 0.5135 .5 \\
\hline 0.66 & 0.53235 & 0.52895 & 0.52551 & 0.52203 & 0.51850 & 0.51493 & 0.51132 & 0.50766 & C. 50395 & $0.5 \mathrm{Cc} 2 \mathrm{C}$ \\
\hline 0.65 & 0.51973 & 0.51020 & 0.51276 & 0.50922 & C. 50563 & C. 50200 & 0.49832 & 0.49460 & 0.49083 & 0.48701 \\
\hline 0.64 & 0.50726 & 0.50373 & 0.50017 & 0.49657 & 0.49292 & $C .48923$ & 0.48550 & C. 48172 & 0.47789 & 0.47402 \\
\hline 0.63 & 0.49494 & 0.49136 & 0.48775 & 0.48409 & 0.48038 & 0.47664 & 0.47285 & 0.46901 & 0.46513 & $0.4612 \mathrm{C}$ \\
\hline 0.62 & 0.48279 & 0.47916 & 0.47549 & 0.47177 & 0.46802 & C. 46422 & 0.46038 & 0.45650 & 0.45256 & 0.44859 \\
\hline 0.61 & 0.47080 & 0.46712 & 0.46340 & 0.45964 & 0.45584 & C. 45159 & $C .44810$ & C. 44417 & 0.44019 & 0.43617 \\
\hline 0.60 & 0.45899 & 0.45526 & 0.45149 & 0.44769 & 0.44384 & 0.43995 & 0.43601 & 0.43204 & C. 42802 & 0.4239 .5 \\
\hline 0.59 & 0.44735 & 0.44358 & 0.43977 & 0.43592 & 0.43203 & 0.42809 & 0.42412 & 0.42010 & 0.41605 & 0.41194 \\
\hline 0.58 & 0.43589 & 0.43208 & 0.42822 & 0.42434 & 0.42041 & C. 41644 & $C .41243$ & 0.40838 & 0.40428 & 0.40014 \\
\hline 0.57 & 0.42461 & 0.42076 & 0.41687 & 0.41295 & 0.40898 & 0.40498 & $c .4 \mathrm{CC} 94$ & 0.35685 & 0.39273 & 0.38856 \\
\hline 0.56 & 0.41352 & $0.40 \times 64$ & 0.40572 & 0.40176 & 0.39776 & C. 39373 & $0.3896 b$ & 0.38554 & 0.38139 & 0.37715 \\
\hline 0.55 & 0.40262 & 0.39870 & 0.39475 & 0.39077 & c. 38674 & c. 32268 & C. 37858 & 0.37444 & 0.37026 & 0.36605 \\
\hline 0.54 & 0.39191 & 0.38797 & 0.38399 & 0.37998 & 0.37593 & 0.37184 & 0.36772 & C. 36356 & 0.35936 & 0.35512 \\
\hline 0.53 & 0.38140 & 0.37743 & 0.37343 & 0.36939 & 0.36532 & 0.36121 & 0.35707 & 0.35289 & 0.34868 & 0.34442 \\
\hline 0.52 & 0.37109 & 0.36710 & 0.36308 & 0.35902 & 0.35493 & C. 35680 & C. $34 t 64$ & 0.34245 & 0.33822 & $02.3339 E$ \\
\hline 0.51 & .0 .36098 & 0.35697 & 0.35293 & 0.34885 & 0.34474 & 0.34061 & 0.33643 & C. 33223 & 0.32799 & 0.32371 \\
\hline 0.50 & 0.35107 & 0.34705 & 0.34299 & 0.33890 & 0.33478 & 0.33063 & 0.32644 & 0.32223 & C. 31798 & $0.3137 \mathrm{C}$ \\
\hline 0.49 & 0.34137 & 0.33133 & 0.33326 & 0.32916 & C.32503 & 0.320 & 0.3166 & 0.31246 & 0.30820 & 0.30392 \\
\hline 0.48 & .0 .33188 & 0.32782 & 0.32374 & 0.31963 & 0.31549 & 0.31133 & 0.30713 & C. 36291 & 0.29866 & 0.29498 \\
\hline 0.47 & 0.32259 & 0.31853 & 0.31444 & 0.31032 & 0.30618 & 0.30201 & 0.29781 & 0.29359 & 0.28934 & 0.28506 \\
\hline 0.46 & 0.31331 & 0.30944 & 0.30535 & 0.30123 & C. 29708 & C. 29291 & 0.28872 & 0.28450 & 0.28025 & 0.27598 \\
\hline 0.45 & 0.30465 & 0.30057 & 0.29647 & 0.29235 & 0.28821 & C. 28404 & C.27585 & C. 27564 & 0.27140 & 0.26714 \\
\hline 0.44 & 0.29599 & 0.29192 & 0.28782 & 0.28370 & 0.27956 & 0.27539 & 0.27121 & 0.26700 & 0.26277 & 0.25852 \\
\hline 0.43 & 0.28755 & 0.28347 & 0.27938 & 0.27526 & 0.27112 & C. $2 \in 6 \subseteq 7$ & 0.26279 & 0.25859 & 0.25438 & 0.25014 \\
\hline 0.42 & 0.27932 & 0.27524 & 0.27115 & 0.26704 & 0.26291 & 0.25877 & $c .254 \in C$ & $c .25 c 41$ & 0.24621 & $0.2419 \mathrm{~s}$ \\
\hline 0.41 & 0.21130 & 0.26123 & 0.26315 & 0.25904 & 0.25492 & 0.25079 & 0.24663 & 0.24246 & 0.23828 & $0.2340 E$ \\
\hline 0.40 & 0.20349 & $0.25 y 43$ & 0.25535 & 0.25126 & $c .24715$ & C. 24303 & c. 238 & 0.23474 & 0.23057 & 0.22639 \\
\hline 0.39 & 0.25590 & 0.25185 & 0.24778 & 0.24370 & 0.23960 & 0.23549 & C. 231 & $c .227$ & 0.22309 & 0.21893 \\
\hline 0.38 & 0.24852 & 0.24448 & 0.24042 & 0.23635 & 0.23227 & 0.22818 & 0.22408 & 0.21596 & 0.21584 & 0.21171 \\
\hline 0.37 & 0.24136 & 0.23733 & 0.23328 & 0.22923 & 0.22516 & 0.22109 & 0.21700 & 0.21291 & 0.20881 & $0.2047 \mathrm{C}$ \\
\hline 0.36 & 0.23440 & 0.23038 & 0.22635 & 0.22231 & 0.21827 & 0.21421 & 0.2101 & C. $2 C \in 08$ & 0.202 & 0.19793 \\
\hline 0.35 & 0.22766 & 0.22365 & 0.2196 & 0.2156 & 0.21159 & 0.20756 & 0.20352 & 0.19547 & 0.19 & C. 19137 \\
\hline 0.34 & 0.22113 & $0.21 / 14$ & 0.21314 & 0.20914 & 0.2051 & C. 20112 & 0.19710 & 0.19308 & 0.18906 & 0.18504 \\
\hline 0.33 & 0.21480 & 0.21083 & 0.20685 & 0.20287 & C.19888 & C. 15489 & C.19C8 & C. $1 \varepsilon \in 91$ & 0.18292 & 0.17893 \\
\hline 0.32 & 0.20869 & 0.20473 & 0.20077 & 0.19681 & 0.19225 & 0.18888 & 0.18492 & 0.18095 & 0.17699 & 0.17303 \\
\hline 0.31 & 0.20278 & 0.19884 & 0.19490 & 0.19096 & $c .18702$ & 0.18308 & 0.17913 & 0.17521 & 0.17128 & 0.16735 \\
\hline 0.30 & 0.19708 & 0.19316 & 0.18924 & 0.18533 & 0.18141 & C. 17749 & 0.173 & 0.16968 & 0.16578 & 0.16188 \\
\hline 0.29 & 0.19159 & 0.18769 & 0.18379 & 0.17989 & 0.17600 & 0.172 & 0.16823 & 0.164 & 0.16048 & 0.15662 \\
\hline 0.28 & 0.18630 & 0.18242 & 0.17854 & 0.17467 & C. 17080 & C. 16694 & 0.16309 & 0.15924 & 0.15540 & 0.15157 \\
\hline 0.27 & 0.18121 & 0.17735 & 0.17350 & 0.16965 & 0.26581 & C. 16157 & C.15815 & c. 15433 & 0.15052 & 0.14673 \\
\hline 0.26 & 0.17633 & 0.17249 & 0.16865 & 0.16483 & 0.16101 & 0.15720 & 0.15341 & C. 14962 & 0.14585 & 0.14205 \\
\hline 0.25 & 0.17164 & 0.16782 & 0.16401 & 0.16021 & 0.15842 & C. 15264 & 0.14837 & 0.14512 & 0.14137 & 0.13765 \\
\hline 0.24 & 0.16715 & 0.16336 & 0.15957 & 0.15579 & 0.15203 & C. 14827 & c.14453 & 0.14081 & 0.13710 & 0.13340 \\
\hline 0.23 & 0.16286 & 0.15909 & 0.15532 & 0.15157 & 0.14783 & 0.14410 & C.1/4c39 & $C .1367 \mathrm{C}$ & 0.13302 & $0.1293 t$ \\
\hline 0.22 & 0.15877 & 0.15502 & 0.15127 & 0.14754 & 0.14383 & 0.14013 & 0.13644 & 0.13278 & 0.12913 & $0.125 .5 \mathrm{C}$ \\
\hline 0.21 & 0.15487 & 0.15114 & 0.14742 & 0.14371 & C. 14002 & c. 13635 & C.13269 & 0.12905 & 0.12544 & 0.12184 \\
\hline 0.20 & 0.15116 & 0.14745 & 0.14375 & 0.14007 & 0.13640 & 0.13276 & $c .12 \$ 13$ & C. 12552 & 0.12193 & 0.11837 \\
\hline
\end{tabular}


TABLES OF CONCENTRATION RATIOS - $c / c_{0}$

\begin{tabular}{|c|c|c|c|c|c|c|c|c|c|c|}
\hline$\frac{0 t / a^{2}}{r / a}$ & 0.080 & 0.079 & 0.078 & c. 077 & 0.076 & 0.075 & 0.074 & 0.073 & 0.072 & 0.071 \\
\hline $\begin{array}{l}1.00 \\
0.99\end{array}$ & 0.98554 & 0.98541 & 0.98527 & 0.98514 & 0.98500 & & 0.98471 & 0.98458 & 0.98441 & \\
\hline 0.98 & 0.97095 & 0.97068 & 0.97041 & 0.57014 & $C .96986$ & 0.96957 & 0.96928 & 0.96899 & 0.96869 & \\
\hline 0.97 & 0.95623 & 0.95583 & 0.95543 & 0.95501 & 0.95459 & $C .95416$ & $c .95373$ & 0.95328 & 0.95283 & 0.95236 \\
\hline 0.96 & 0.94140 & 0.94087 & 0.94032 & 0.93977 & 0.93921 & 0.93864 & 0.93805 & 0.93746 & $\begin{array}{r}0.93685 \\
0.92077\end{array}$ & $\begin{array}{l}0.93623 \\
0.91999\end{array}$ \\
\hline 0.95 & 0.92647 & 0.92580 & 0.92512 & 0.52443 & 0.92372 & 0.92301 & 0.92227 & 0.92153 & $\begin{array}{l}0.92077 \\
0.90459\end{array}$ & $\begin{array}{l}0.91999 \\
0.90366\end{array}$ \\
\hline 0.94 & 0.91144 & 0.91064 & 0.90982 & 0.90899 & 0.90815 & C. SC728 & $\begin{array}{l}0.96640 \\
0.89645\end{array}$ & $\begin{array}{l}0.90551 \\
C .8854 \mathrm{C}\end{array}$ & $\begin{array}{l}0.90459 \\
0.88833\end{array}$ & $\begin{array}{l}0.90366 \\
0.88724\end{array}$ \\
\hline $\begin{array}{l}0.93 \\
0.92\end{array}$ & 0.89634 & $\begin{array}{l}0.89540 \\
0.88009\end{array}$ & $\begin{array}{l}0.89445 \\
0.87900\end{array}$ & $\begin{array}{l}0.89348 \\
0.87789\end{array}$ & $\begin{array}{l}0.89249 \\
0.87676\end{array}$ & $\begin{array}{l}0.89148 \\
0.87561\end{array}$ & $\begin{array}{l}0.89645 \\
0.87443\end{array}$ & $\begin{array}{l}C .8854 C \\
0.87323\end{array}$ & 0.87201 & $\begin{array}{r}0.88784 \\
0687077\end{array}$ \\
\hline $\begin{array}{l}0.92 \\
0.91\end{array}$ & 0.88593 & 0.86472 & 0.86350 & c. 86225 & & C. 85968 & 0.85835 & 0.85701 & 0.85563 & 0.85423 \\
\hline 0.90 & 0.85065 & 0.84931 & 0.84795 & 0.84656 & 0.84514 & 0.84370 & C. 84224 & c. $84 C 74$ & 0.83921 & 0.83766 \\
\hline 0.89 & 0.83533 & 0.83386 & 0.83236 & 0.83083 & 0.82928 & 0.82770 & 0.82608 & $\begin{array}{l}0.82444 \\
0.80813\end{array}$ & $\begin{array}{l}0.82277 \\
0.80630\end{array}$ & $\begin{array}{l}0.82106 \\
0.80444\end{array}$ \\
\hline 0.88 & & 0.81838 & 0.81675 & 0.81509 & 0.81339 & $\begin{array}{l}0.81167 \\
0.79564\end{array}$ & $\begin{array}{l}0.80592 \\
0.79374\end{array}$ & $\begin{array}{l}0.80813 \\
c .79181\end{array}$ & 0.78984 & 0.78783 \\
\hline $\begin{array}{l}0.87 \\
0.86\end{array}$ & $\begin{array}{l}0.80462 \\
0.78926\end{array}$ & $\begin{array}{l}0.80289 \\
0.78740\end{array}$ & $\begin{array}{l}0.80112 \\
0.78550\end{array}$ & $\begin{array}{l}0.79933 \\
0.78357\end{array}$ & $\begin{array}{l}0.79750 \\
0.78161\end{array}$ & 0.77961 & $\begin{array}{l}0.79374 \\
0.77757\end{array}$ & 0.77549 & 0.77338 & 0677122 \\
\hline 0.85 & 0.77390 & 0.77191 & 0.76988 & 0.76782 & 0.76573 & C. 76359 & 0.76142 & 0.75920 & 0.75694 & 0.75464 \\
\hline 0.84 & 0.75856 & 0.75644 & 0.75429 & 0.75210 & $0.74 y 87$ & 0.74760 & 0.74529 & C. 74294 & 0.74054 & 0.73810 \\
\hline 0.83 & 0.74324 & 0.74100 & 0.73873 & 0.73641 & 0.73405 & 0.73165 & 0.72921 & 0.72672 & 0.72419 & 0.72161 \\
\hline 0.82 & 0.72796 & 0.72560 & 0.72321 & 0.72076 & 0.71828 & 0.71575 & 0.71318 & 0.71056 & 0.70790 & 0.70518 \\
\hline 0.81 & 0.71273 & 0.71026 & 0.70774 & 0.70518 & 0.70257 & C.ts\$\$2 & $c .69722$ & C. $6 \$ 447$ & 0.69167 & 0.68882 \\
\hline 0.80 & 0.69756 & 0.69497 & & 0.68965 & 0.68693 & 0.68415 & 0.68133 & 0.67846 & $\begin{array}{l}0.67553 \\
0.65949\end{array}$ & $\begin{array}{l}0.67256 \\
0.6563 \ell\end{array}$ \\
\hline $\begin{array}{l}0.78 \\
0.77\end{array}$ & $\begin{array}{l}0.66743 \\
0.65249\end{array}$ & 0.64957 & $\begin{array}{l}0.66175 \\
0.64660\end{array}$ & 0.64358 & 0.64052 & 0.63740 & 0.63423 & 0.63100 & 0.62772 & 0.62438 \\
\hline 0.76 & 0.63765 & 0.63462 & 0.63155 & 0.62843 & 0.62525 & 0.62203 & 0.61875 & 0.61541 & 0.61202 & 0.60857 \\
\hline 0.75 & 0.62291 & 0.61979 & 0.61661 & 0.61339 & 0.61011 & C. $E C \in 78$ & 0.60340 & 0.59995 & 0.59645 & 0.59289 \\
\hline 0.74 & 0.60829 & 0.60507 & 0.60180 & 0.59847 & 0.59510 & 0.59167 & C. $58 \varepsilon 18$ & C. 58464 & 0.58104 & 0.57737 \\
\hline 0.73 & 0.59379 & 0.59047 & 0.58711 & 0.58369 & 0.58022 & 0.57670 & 0.57311 & 0.56948 & 0.56578 & 0.56202 \\
\hline 0.72 & 0.57942 & 0.57602 & 0.57256 & 0.56905 & 0.56549 & C. 56188 & 0.55820 & 0.55447 & 0.55068 & 0.54682 \\
\hline 0.71 & 0.56519 & 0.56170 & 0.55816 & 0.55457 & 0.55092 & 0.54722 & C. 54346 & C. 53564 & 0.53576 & 0.53181 \\
\hline 0.70 & 0.55111 & 0.54754 & 0.54392 & 0.54024 & 0.53651 & 0.53272 & 0.52888 & 0.52498 & 0.52102 & 0.51699 \\
\hline 0.69 & 0.53718 & 0.53353 & 0.52983 & 0.52608 & 0.52227 & 0.51841 & 0.51449 & 0.51051 & 0.50647 & 0.50236 \\
\hline 0.68 & 0.52341 & 0.51969 & 0.51592 & 0.51209 & 0.50821 & 0.50428 & C. $5 c c 28$ & C. 49623 & 0.49211 & 0.48794 \\
\hline 0.67 & 0.50981 & 0.50602 & 0.50218 & 0.49829 & 0.49434 & 0.49033 & 0.48627 & 0.48215 & 0.47797 & 0.47372 \\
\hline 0.66 & 0.49639 & 0.49254 & 0.48863 & 0.48467 & 0.48065 & 0.47659 & 0.47246 & 0.46827 & 0.46403 & 0.45972 \\
\hline 0.65 & 0.48315 & 0.47923 & 0.47526 & 0.47124 & 0.46717 & $C .463 C 4$ & $C .45 E \varepsilon 6$ & C. 45461 & 0.45031 & 0.44595 \\
\hline 0.64 & 0.47009 & 0.46612 & 0.46209 & 0.45802 & 0.45389 & 0.44971 & 0.44541 & 0.44117 & 0.43682 & $0.4324 \mathrm{C}$ \\
\hline 0.63 & 0.45723 & 0.45320 & 0.44913 & $0.4450 \mathrm{C}$ & 0.44082 & 0.43659 & 0.43230 & 0.42795 & 0.42355 & 0.41905 \\
\hline 0.62 & 0.44456 & 0.44049 & 0.43636 & 0.43219 & 0.42796 & $c .42369$ & $c .41535$ & 0.41496 & 0.41052 & 0.40602 \\
\hline 0.61 & 0.43210 & 0.42798 & 0.42381 & 0.41960 & 0.41533 & 0.41101 & 0.40664 & 0.40221 & 0.39773 & 0.39319 \\
\hline 0.60 & 0.41984 & 0.41568 & 0.41148 & 0.40722 & C. 40292 & 0.39856 & 0.39415 & 0.38969 & 0.38518 & 0.38061 \\
\hline 0.59 & 0.40779 & 0.40360 & 0.39936 & 0.39507 & 0.39073 & c. 38634 & 0.38191 & 0.37742 & 0.37288 & 0.36828 \\
\hline 0.58 & 0.39596 & 0.39174 & 0.38746 & 0.38314 & 0.37878 & 0.37437 & $c .36590$ & $c .36539$ & 0.36083 & $0.3562 \mathrm{I}$ \\
\hline 0.57 & 0.38435 & 0.38009 & 0.37580 & 0.37145 & 0.36706 & 0.36263 & 0.35814 & 0.35361 & 0.34903 & $013444 \mathrm{C}$ \\
\hline 0.56 & 0.37296 & 0.36868 & 0.36436 & 0.15999 & 0.35558 & C. 35113 & C. 344663 & 0.34208 & 0.33749 & 0.33284 \\
\hline 0.55 & 0.36179 & 0.35749 & 0.35315 & 0.34877 & 0.34434 & 0.33987 & 0.33536 & $C .33 C 8 C$ & 0.32620 & 0.32155 \\
\hline 0.54 & 0.35085 & 0.34653 & 0.34216 & 0.33778 & 0.33335 & 0.32887 & 0.32435 & 0.31578 & 0.31518 & 0.31053 \\
\hline 0.53 & 0.34014 & 0.33581 & 0.33144 & 0.32704 & 0.32259 & c. 31811 & 0.31358 & 0.30902 & 0.30441 & 0.29977 \\
\hline 0.52 & 0.32965 & 0.32532 & 0.32094 & 0.31653 & 0.31209 & $0.307 \in 0$ & C. $3 \mathrm{C} 3 \mathrm{C} 8$ & C. 2SE5I & 0.29391 & 0.28927 \\
\hline 0.51 & 0.31941 & 0.31506 & 0.31069 & 0.30627 & 0.30183 & 0.29734 & 0.29282 & 0.28826 & 0.28367 & 0.27904 \\
\hline 0.50 & 0.30939 & 0.30505 & 0.30067 & c. 29626 & 0.29181 & 0.28734 & 0.28282 & 0.27828 & 0.27370 & 0.26988 \\
\hline 0.49 & 0.29961 & 0.29527 & 0.29089 & 0.28649 & 0.28205 & 0.27758 & $0.2736 \mathrm{Cg}$ & C. $26 E 55$ & 0.26398 & 0.25939 \\
\hline 0.48 & 0.29007 & 0.28573 & 0.28136 & 0.27696 & 0.27253 & 0.26808 & 0.26359 & 0.25908 & 0.25453 & 0.24996 \\
\hline 0.47 & 0.28076 & 0.27643 & 0.27207 & 0.26768 & 0.26327 & 0.25883 & 0.25436 & 0.24986 & 0.24534 & 0.24079 \\
\hline 0.46 & 0.27169 & 0.26736 & 0.26302 & 0.25865 & 0.25425 & 0.2498 & $c .24538$ & C. 24091 & 0.23641 & 0.23189 \\
\hline 0.45 & 0.26285 & & 0.25421 & 0.24986 & 0.24548 & 0.24108 & 0.23666 & 0.232 & C. 22774 & C. 22326 \\
\hline 0.44 & 0.25425 & 0.24996 & 0.24564 & 0.24131 & 0.23695 & 0.23258 & 0.22818 & 0.22377 & 0.21933 & $0.2148 \varepsilon$ \\
\hline 0.43 & 0.24589 & 0.24161 & 0.23732 & 0.23301 & 0.22868 & C. 22433 & 0.21596 & C.21558 & 0.21118 & 0.20676 \\
\hline 0.42 & 0.23776 & 0.23350 & 0.22923 & 0.22494 & 0.22064 & 0.21632 & 0.21199 & 0.20764 & 0.20328 & $0.1989 \mathrm{C}$ \\
\hline 0.41 & 0.22986 & 0.22563 & 0.22138 & 0.21712 & 0.21285 & 0.20856 & 0.20426 & 0.19995 & 0.19563 & 0.19136 \\
\hline 0.40 & 0.22220 & 0.21799 & 0.21377 & 0.20954 & 0.20530 & C. 20105 & C. 19678 & 0.19251 & 0.18823 & 0.18394 \\
\hline 0.39 & 0.21476 & 0.21058 & 0.20639 & 0.20220 & 0.19799 & 0.19377 & 0.185 & $c .18531$ & 0.18108 & 0.17683 \\
\hline 0.38 & 0.20756 & 0.20341 & 0.19925 & 0.19508 & 0.19091 & 0.18673 & 0.18255 & 0.17836 & 0.17417 & 0.16991 \\
\hline 0.37 & 0.20059 & 0.19647 & 0.19234 & 0.18821 & 0.18407 & C. 1799 & 0.17579 & 0.17 & 0.16750 & 0.16335 \\
\hline 0.36 & 0.19384 & $0.18 y 75$ & 0.18566 & 0.18156 & 0.17746 & 0.17336 & C. $1 \in \$ 26$ & $c .16516$ & 0.16107 & 0.15697 \\
\hline 0.35 & 0.18732 & 0.18326 & 0.17920 & 0.17514 & 0.17108 & 0.16703 & 0.16297 & 0.15892 & 0.15487 & 0.15082 \\
\hline 0.34 & 0.18102 & 0.17699 & 0.17297 & 0.16895 & 0.16493 & $0.1 \in 092$ & 0.156 & 0.15290 & 0.1489 .0 & 0.14491 \\
\hline 0.33 & 0.17494 & 0.17095 & 0.16696 & 0.16298 & 0.15900 & 0.15503 & C.151C7 & C. 14711 & 0.14317 & 0.1392 .3 \\
\hline 0.32 & 0.16907 & 0.16512 & 0.16117 & 0.15723 & 0.15330 & 0.14937 & 0.14545 & 0.14155 & 0.13765 & 0.13377 \\
\hline 0.31 & 0.16343 & 0.15451 & 0.15560 & $0.1517 \mathrm{C}$ & 0.14781 & C. 14393 & 0.140 & 0.13620 & 0.13236 & 0.12853 \\
\hline 0.30 & 0.15799 & 0.15411 & 0.15024 & 0.14638 & 0.14253 & C. 13870 & 0.13488 & C. 13107 & 0.12728 & 0.12350 \\
\hline 0.29 & 0.15277 & 0.14893 & 0.14510 & 0.14128 & 0.13747 & 0.13368 & 0.12991 & 0.12615 & 0.12241 & 0.11869 \\
\hline 0.28 & 0.14776 & 0.14395 & 0.14016 & 0.13638 & c. 13262 & 0.12888 & 0.125 & 0.12144 & 0.11775 & 0.11409 \\
\hline 0.27 & 0.14295 & 0.13918 & 0.13543 & 0.13169 & 0.12797 & c. 12427 & 0.120 & c. $11 \in 94$ & 0.11330 & 0.10969 \\
\hline 0.26 & 0.13834 & 0.13461 & 0.13090 & 0.12720 & 0.12353 & 0.11987 & 0.11624 & $0.11 \times 63$ & 0.10905 & 0.10545 \\
\hline 0.25 & 0.13394 & 0.13024 & 0.12657 & 0.12292 & C.11928 & 0.11567 & 0.11209 & 0.10853 & 0.10500 & 0.10145 \\
\hline & 0.12973 & 0.12607 & 0.12244 & 0.11883 & 0.11524 & C. 11167 & $0.1 C 813$ & C. 10462 & 0.10113 & 0.09768 \\
\hline 0.23 & 0.12572 & 0.12210 & 0.11850 & 0.11493 & 0.11138 & 0.10786 & 0.10436 & 0.10090 & 0.09746 & 0.09406 \\
\hline 0.22 & 0.12190 & 0.11832 & 0.11476 & 0.11122 & 0.10771 & C. 10424 & 0.10079 & 0.09737 & 0.09398 & 0.09063 \\
\hline 0.21 & 0.11827 & 0.11472 & 0.11120 & 0.10770 & 0.10424 & C. $1 C C B C$ & 0.09739 & 0.09402 & 0.09068 & 0.08738 \\
\hline 0.20 & 0.11483 & 0.11132 & 0.10783 & 0.10437 & 0.10094 & 0.09755 & c.cs418 & $\operatorname{cosc} 85$ & 0.08756 & $0 . C 843 C$ \\
\hline
\end{tabular}


TABLES OF CONCENTRATION RATIOS $-c / c_{0}$

\begin{tabular}{|c|c|c|c|c|c|c|c|c|c|c|}
\hline$\frac{D t / a^{2}}{r / a}$ & 0.070 & 0.069 & 0.068 & 0.067 & 0.066 & 0.065 & 0.064 & 0.063 & 0.062 & 0.001 \\
\hline $\begin{array}{l}1.00 \\
0.99\end{array}$ & 0.98410 & 0.98394 & 0.98378 & 0.98361 & 0.98344 & 0.98327 & 0.98309 & 0.98291 & 0.98272 & $\begin{array}{l}0.96253 \\
0.96491\end{array}$ \\
\hline $\begin{array}{l}0.98 \\
0.97\end{array}$ & $\begin{array}{l}0.96806 \\
0.75189\end{array}$ & $\begin{array}{l}0.96774 \\
0.95141\end{array}$ & $\begin{array}{l}0.96741 \\
0.95091\end{array}$ & $\begin{array}{l}0.96708 \\
0.95041\end{array}$ & $\begin{array}{l}c .96674 \\
0.94989\end{array}$ & $\begin{array}{l}0.96639 \\
c .94937\end{array}$ & $\begin{array}{l}0.96603 \\
0.94883\end{array}$ & $\begin{array}{l}0.96566 \\
0.94828\end{array}$ & $\begin{array}{l}0.96529 \\
0.94772\end{array}$ & $\begin{array}{l}0.96491 \\
0.94714\end{array}$ \\
\hline 0.96 & 0.93560 & 0.93495 & 0.93429 & 0.93362 & 0.93293 & 0.93223 & 0.93151 & $c .93677$ & 0.93002 & 0.92924 \\
\hline $\begin{array}{l}0.95 \\
0.94\end{array}$ & $\begin{array}{l}0.91920 \\
0.70270\end{array}$ & $\begin{array}{l}0.91139 \\
0.90173\end{array}$ & $\begin{array}{l}0.91756 \\
0.90074\end{array}$ & $\begin{array}{l}0.91672 \\
0.89973\end{array}$ & $\begin{array}{l}0.91586 \\
0.89869\end{array}$ & $\begin{array}{l}0.91497 \\
\text { C. 89763 }\end{array}$ & $\begin{array}{l}0.91407 \\
0.89655\end{array}$ & $\begin{array}{l}0.91315 \\
0.89544\end{array}$ & $\begin{array}{l}0.91221 \\
0.89431\end{array}$ & $\begin{array}{l}0.91124 \\
0.89315\end{array}$ \\
\hline 0.93 & 0.88613 & 0.88500 & 0.88384 & 0.88265 & 0.88145 & 0.88021 & 0.87895 & C.87765 & 0.87633 & 0.87498 \\
\hline 0.92 & 0.86949 & 0.86880 & 0.86687 & 0.86552 & 0.86414 & 0.86273 & 0.86128 & 0.85981 & 0.85829 & 0.85675 \\
\hline 0.91 & 0.85280 & 0.85134 & 0.84985 & 0.84833 & C.84678 & C. 84519 & 0.84357 & 0.84191 & 0.84021 & 0.83848 \\
\hline 0.90 & 0.83607 & 0.83445 & $\begin{array}{l}0.83280 \\
0.81572\end{array}$ & $\begin{array}{l}0.83111 \\
0.81387\end{array}$ & $\begin{array}{l}0.82939 \\
0.81198\end{array}$ & $\begin{array}{l}0.82763 \\
0.81005\end{array}$ & $\begin{array}{l}0.82583 \\
0.80807\end{array}$ & $\begin{array}{l}\text { C.823.99 } \\
0.80605\end{array}$ & $\begin{array}{l}0.82211 \\
0.80399\end{array}$ & $\begin{array}{l}0.82018 \\
0.80188\end{array}$ \\
\hline $\begin{array}{l}0.89 \\
0.88\end{array}$ & $\begin{array}{l}0.81932 \\
0.30255\end{array}$ & $\begin{array}{l}0.81754 \\
0.80061\end{array}$ & $\begin{array}{l}0.81572 \\
0.79864\end{array}$ & $\begin{array}{l}0.81387 \\
0.79662\end{array}$ & $\begin{array}{l}0.81198 \\
0.79457\end{array}$ & $\begin{array}{l}0.81905 \\
c .79246\end{array}$ & 0.79032 & 0.78812 & 0.78588 & 0.78358 \\
\hline 0.87 & 0.78578 & 0.78369 & 0.78156 & 0.77938 & 0.77716 & $\begin{array}{l}0.77489 \\
0.75735\end{array}$ & $\begin{array}{l}0.77257 \\
0.75486\end{array}$ & $c .77 c 21$ & 0.76778 & $\begin{array}{l}0.76531 \\
0.74708\end{array}$ \\
\hline 0.86 & 0.76903 & 0.76678 & $\begin{array}{l}0.76450 \\
0.74747\end{array}$ & 0.76216 & $\begin{array}{l}0.75978 \\
0.74244\end{array}$ & $\begin{array}{l}0.75735 \\
\text { c. } 73985\end{array}$ & $\begin{array}{l}0.75486 \\
0.73720\end{array}$ & $\begin{array}{l}0.75233 \\
0.73449\end{array}$ & $\begin{array}{l}0.74973 \\
0.73173\end{array}$ & $\begin{array}{l}0.74708 \\
0.72890\end{array}$ \\
\hline $\begin{array}{l}0.85 \\
0.84\end{array}$ & $\begin{array}{l}0.75230 \\
0.73561\end{array}$ & $\begin{array}{l}0.74991 \\
0.73308\end{array}$ & $\begin{array}{l}0.74747 \\
0.73049\end{array}$ & $\begin{array}{l}0.74498 \\
0.72785\end{array}$ & $\begin{array}{l}0.74244 \\
0.72515\end{array}$ & $\begin{array}{l}C .13224 C \\
C .7224\end{array}$ & 0.71959 & C. $71 \in 73$ & 0.71379 & 0.71080 \\
\hline 0.83 & 0.71898 & 0.71630 & 0.71356 & 0.71078 & 0.70793 & 0.70503 & 0.70206 & 0.69904 & 0.69594 & 0.69278 \\
\hline 0.82 & 0.70241 & 0.69959 & 0.69671 & 0.69378 & 0.69079 & 0.68774 & 0.68462 & 0.68144 & 0.67819 & 0.67487 \\
\hline 0.81 & 0.60592 & 0.68297 & 0.67995 & 0.67688 & 0.67374 & c. 67055 & 0.66728 & 0.66395 & 0.66055 & 0.65708 \\
\hline 0.80 & 0.66952 & 0.66643 & 0.66328 & 0.66007 & $\begin{array}{r}0.65680 \\
.63998\end{array}$ & $\begin{array}{l}0.65346 \\
0.63651\end{array}$ & $\begin{array}{l}0.65006 \\
0.63297\end{array}$ & $\begin{array}{l}0.64658 \\
0.62935\end{array}$ & $\begin{array}{l}0.64304 \\
0.62560\end{array}$ & $\begin{array}{l}0.63942 \\
0.6219 c\end{array}$ \\
\hline 0.79 & 0.65322 & 0.65001 & & $\begin{array}{l}0.64339 \\
0.42683\end{array}$ & $\begin{array}{l}\text { C. } 63998 \\
0.62329\end{array}$ & $\begin{array}{l}0.63651 \\
\text { c. } 61969\end{array}$ & $\begin{array}{l}0.63297 \\
0.616 \mathrm{CL}\end{array}$ & $\begin{array}{l}0.62935 \\
0.61227\end{array}$ & 0.62560 & $\begin{array}{l}0.62196 \\
0.60454\end{array}$ \\
\hline $\begin{array}{l}0.78 \\
0.77\end{array}$ & $\begin{array}{l}0.63704 \\
0.62098\end{array}$ & $\begin{array}{l}0.63370 \\
0.61752\end{array}$ & $\begin{array}{l}0.63029 \\
0.61399\end{array}$ & $\begin{array}{l}0.82683 \\
0.61040\end{array}$ & 0.60675 & 0.60302 & 0.59522 & C.59534 & 0.59139 & 0.58736 \\
\hline 0.76 & 0.60505 & 0.60148 & 0.59784 & 0.59413 & 0.59036 & 0.58651 & 0.58259 & 0.57859 & 0.57452 & $0: 5703 t$ \\
\hline 0.75 & 0.58927 & 0.58559 & 0.58184 & 0.57802 & 0.57413 & C. 57017 & 0.56614 & 0.56202 & 0.55783 & 0.5535 .6 \\
\hline 0.74 & 0.57365 & 0.56986 & 0.56600 & 0.56208 & 0.55808 & 0.55402 & 0.54587 & C. 54565 & 0.54135 & 0.53697 \\
\hline 0.73 & 0.55819 & 0.55430 & 0.55035 & 0.54632 & 0.54223 & 0.53806 & 0.53381 & 0.52949 & 0.52508 & C. $5206 \mathrm{C}$ \\
\hline 0.72 & 0.54291 & 0.53892 & 0.53487 & 0.53075 & 0.52656 & $0.5223 \mathrm{C}$ & C.51796 & C. 51354 & 0.50904 & 0.50445 \\
\hline 0.71 & 0.52781 & 0.52374 & 0.51960 & 0.51539 & 0.51111 & 0.50675 & 0.50232 & C. 49781 & 0.49322 & 0.48855 \\
\hline 0.70 & 0.51290 & 0.50875 & 0.50452 & 0.50023 & 0.49587 & 0.49143 & 0.48692 & 0.48232 & 0.47765 & $0.4729 \mathrm{C}$ \\
\hline 0.69 & 0.49820 & 0.49396 & 0.48966 & 0.48529 & $c .48085$ & C. 47634 & 0.47175 & 0.46708 & 0.46233 & 0.45750 \\
\hline 0.68 & 0.48370 & 0.47439 & 0.47502 & 0.47058 & 0.46607 & 0.46148 & $C .45 t \varepsilon 2$ & $C .45209$ & 0.44727 & 0.44237 \\
\hline 0.67 & 0.46942 & 0.46504 & 0.46061 & 0.45610 & 0.45152 & 0.44687 & 0.44215 & 0.43735 & 0.43247 & 0.42752 \\
\hline 0.66 & 0.45536 & 0.45092 & 0.44642 & 0.44186 & $c .43722$ & C. 43252 & $C .42774$ & 0.42288 & 0.41795 & 0.41294 \\
\hline 0.65 & 0.44152 & 0.43704 & 0.43248 & 0.42786 & 0.42318 & 0.41842 & 0.41359 & $C .40 \varepsilon 65$ & 0.40371 & 0.39865 \\
\hline 0.64 & 0.42793 & 0.42339 & 0.41879 & 0.41412 & 0.40939 & 0.40458 & 0.39971 & 0.39477 & 0.38975 & 0.38465 \\
\hline 0.63 & 0.41457 & 0.40999 & 0.40534 & 0.40664 & C. 39586 & C. 39102 & $0.38 \in 11$ & 0.38113 & 0.37608 & 0.37095 \\
\hline 0.62 & 0.40146 & 0.39684 & 0.39216 & 0.38741 & 0.38260 & 0.37773 & c. 37279 & C. 36778 & 0.36270 & 0.35755 \\
\hline 0.61 & 0.38860 & 0.38394 & 0.37923 & 0.37446 & 0.36962 & 0.36472 & 0.35975 & 0.35472 & 0.34962 & $0.3444 t$ \\
\hline 0.60 & 0.37599 & 0.37131 & 0.36657 & 0.36177 & C. 35691 & 0.35199 & 0.34701 & 0.34196 & 0.33685 & 0.33167 \\
\hline 0.59 & 0.36364 & 0.35893 & 0.35417 & 0.34936 & C. 34448 & 0.33955 & 0.33455 & C. 32549 & 0.32438 & 0.31919 \\
\hline 0.58 & 0.35154 & 0.34682 & 0.34205 & 0.33722 & 0.33233 & 0.32739 & 0.32239 & 0.31733 & 0.31221 & 0.30703 \\
\hline 0.57 & 0.33972 & 0.33498 & 0.33020 & $0.3253 t$ & c. 32047 & c. 31532 & 0.31052 & 0.30547 & 0.30035 & $0.29,518$ \\
\hline 0.56 & 0.32815 & 0.32341 & 0.31863 & 0.31379 & 0.30889 & $6.3 C \geq 95$ & 0.29855 & C. 29391 & 0.28880 & 0.28364 \\
\hline 0.55 & 0.31686 & 0.31212 & 0.30733 & 0.30249 & 0.29760 & 0.29267 & 0.28768 & 0.28265 & 0.27756 & 0.27243 \\
\hline 0.54 & 0.30583 & 0.30109 & 0.29631 & 0.29148 & $c .28660$ & C. 28168 & 0.27671 & 0.27170 & 0.26663 & 0.26152 \\
\hline 0.53 & 0.29508 & 0.29034 & 0.28557 & 0.28075 & 0.27589 & c. 27099 & c. $266 \mathrm{C4}$ & 0.26105 & 0.25601 & 0.25093 \\
\hline 0.52 & 0.28459 & 0.27987 & 0.27511 & 0.27031 & 0.26547 & 0.26059 & $c .25566$ & 0.25070 & 0.24570 & 0.24065 \\
\hline 0.51 & 0.27438 & 0.26967 & 0.26493 & 0.26015 & C. 25533 & C. 25048 & 0.24559 & 0.24066 & 0.23569 & 0.23069 \\
\hline 0.50 & 0.26443 & 0.25975 & 0.25503 & 0.25027 & 0.24549 & C. $24 \mathrm{C} \in 6$ & $C .23581$ & 0.23092 & 0.22599 & 0.22103 \\
\hline 0.49 & 0.25476 & 0.25010 & 0.24540 & 0.24068 & 0.23593 & 0.23114 & $c .22632$ & $c .22147$ & 0.21659 & 0.21169 \\
\hline 0.48 & 0.24535 & 0.24072 & 0.23606 & 0.23137 & 0.22665 & 0.22190 & 0.21713 & 0.21233 & 0.20750 & 0.20264 \\
\hline 0.47 & 0.23622 & 0.23162 & 0.22699 & 0.22234 & 0.21766 & C. 21295 & C. $2 \mathrm{cg} 23$ & 0.20347 & 0.19870 & 0.19390 \\
\hline 0.46 & 0.22135 & 0.22278 & 0.21819 & 0.2135 & 0.20894 & 0.20429 & $c .19 g \in 1$ & C.15491 & 0.19019 & 0.18546 \\
\hline 0.45 & 0.21875 & 0.21422 & 0.20967 & 0.20510 & 0.20051 & 0.19590 & 0.19128 & 0.18664 & 0.18198 & 0.17731 \\
\hline 0.44 & 0.21041 & 0.20592 & 0.20141 & 0.19689 & 0.19235 & C. 18 & C.18323 & $0.17 \varepsilon$ & 0.17405 & 0.16945 \\
\hline 0.43 & 0.20233 & 0.19789 & 0.19343 & 0.18895 & 0.18447 & 0.17997 & 0.17546 & C. $17 C 94$ & 0.16641 & 0.16188 \\
\hline 0.42 & 0.19451 & 0.19011 & 0.18570 & 0.18128 & 0.17685 & 0.17241 & 0.16796 & $c: 16251$ & C. 15905 & $0.1545 \varepsilon$ \\
\hline 0.41 & 0.18695 & 0.18260 & 0.17824 & 0.17387 & C. 16950 & c. 16512 & 0.16073 & 0.15635 & 0.15196 & 0.14757 \\
\hline 0.40 & 0.17964 & 0.17534 & 0.17103 & 0.16672 & 0.16241 & $C .158 C 9$ & 0.15377 & C. 14545 & 0.14514 & 0.14082 \\
\hline 0.39 & 0.17259 & 0.16034 & 0.16408 & 0.15983 & 0.15557 & 0.15132 & 0.14707 & 0.14282 & 0.13858 & 0.13434 \\
\hline 0.38 & 0.16578 & 0.16158 & 0.15738 & 0.15319 & 0.14900 & 0.14481 & 0.14062 & 0.13645 & 0.13228 & 0.12812 \\
\hline 0.37 & 0.15721 & 0.15507 & 0.15093 & 0.14679 & 0.14267 & C.1385 & C. 13443 & $c .13 c 33$ & 0.12 & 0.12216 \\
\hline 0.36 & 0.15288 & 0.14880 & 0.14472 & 0.1406 & 0.136 & & 0.12848 & 0.12446 & 0.12044 & 0.11644 \\
\hline 0.35 & 0.14679 & 0.14276 & 0.13874 & c. 13473 & 0.13074 & c. 12 & 0.12238 & 0.11883 & 0.1148 & 0.11097 \\
\hline 0.34 & 0.14093 & 0.13676 & 0.13300 & 0.12906 & 0.12513 & C. 12121 & $c .11731$ & 0.11343 & 0.10958 & 0.10574 \\
\hline 0.33 & 0.13530 & 0.13139 & 0.12749 & 0.12361 & 0.11975 & 0.115 & 0.11208 & 0.10827 & 0.10449 & 0.10074 \\
\hline 0.32 & 0.12990 & 0.12605 & 0.12221 & 0.11839 & 0.11459 & & & 0.10334 & 0.04964 & 0.09596 \\
\hline 0.31 & 0.12472 & 0.12092 & 0.11715 & 0.11339 & 0.10966 & C. $1 C 596$ & C.1C228 & 0.09862 & 0.09500 & 0.09141 \\
\hline 0.30 & 0.11975 & 0.11601 & 0.11230 & 0.10861 & 0.10495 & 0.10131 & $0 . c 5770$ & c.cs4 12 & 0.090 & 0.08707 \\
\hline 0.29 & 0.11499 & 0.11132 & 0.10767 & 0.10404 & 0.10045 & 0.09688 & 0.09334 & 0.08984 & 0.08637 & 0.08293 \\
\hline 0.28 & 0.11045 & 0.10683 & 0.10324 & 0.65968 & 0.09615 & C.c9265 & 0.08918 & 0.08575 & 0.08236 & 0.07900 \\
\hline 0.27 & 0.10610 & 0.10255 & 0.09902 & 0.09552 & 0.04205 & 0.08862 & C. 08522 & C.08187 & 0.07855 & 0.07527 \\
\hline 0.26 & 0.10196 & 0.09846 & 0.09499 & 0.09156 & 0.08815 & 0.08479 & 0.08146 & 0.07817 & 0.07493 & 0.07172 \\
\hline 0.25 & 0.09802 & 0.09457 & 0.09116 & 0.08775 & 0.08445 & c. 08115 & c.07709 & 0.07467 & 0.07150 & 0.06837 \\
\hline 0.24 & 0.09426 & 0.09087 & 0.08752 & 0.08421 & 0.08093 & 0.07769 & C.C.745C & C. 07135 & 0.06825 & 0.06519 \\
\hline 0.23 & 0.09069 & 0.08736 & 0.08407 & 0.08081 & 0.07759 & 0.07442 & 0.07129 & 0.06821 & 0.06517 & 0.06218 \\
\hline 0.22 & 0.38731 & 0.08403 & 0.08079 & 0.07759 & 0.07444 & 0.07132 & 0.06826 & 0.06524 & 0.06227 & 0505935 \\
\hline 0.21 & 0.08411 & 0.08088 & 0.07770 & 0.07455 & 0.07145 & $c . c \in e 4 C$ & c.ces39 & C. 06244 & $c .05953$ & 0.05668 \\
\hline 0.20 & 0.08108 & 0.07791 & 0.07477 & 0.07168 & 0.06864 & 0.06564 & 0.06270 & 0.05980 & 0.05696 & 0.05417 \\
\hline
\end{tabular}


TABLES OF CONCENTRATION RATIOS - $\mathrm{c} / \mathrm{c}_{0}$

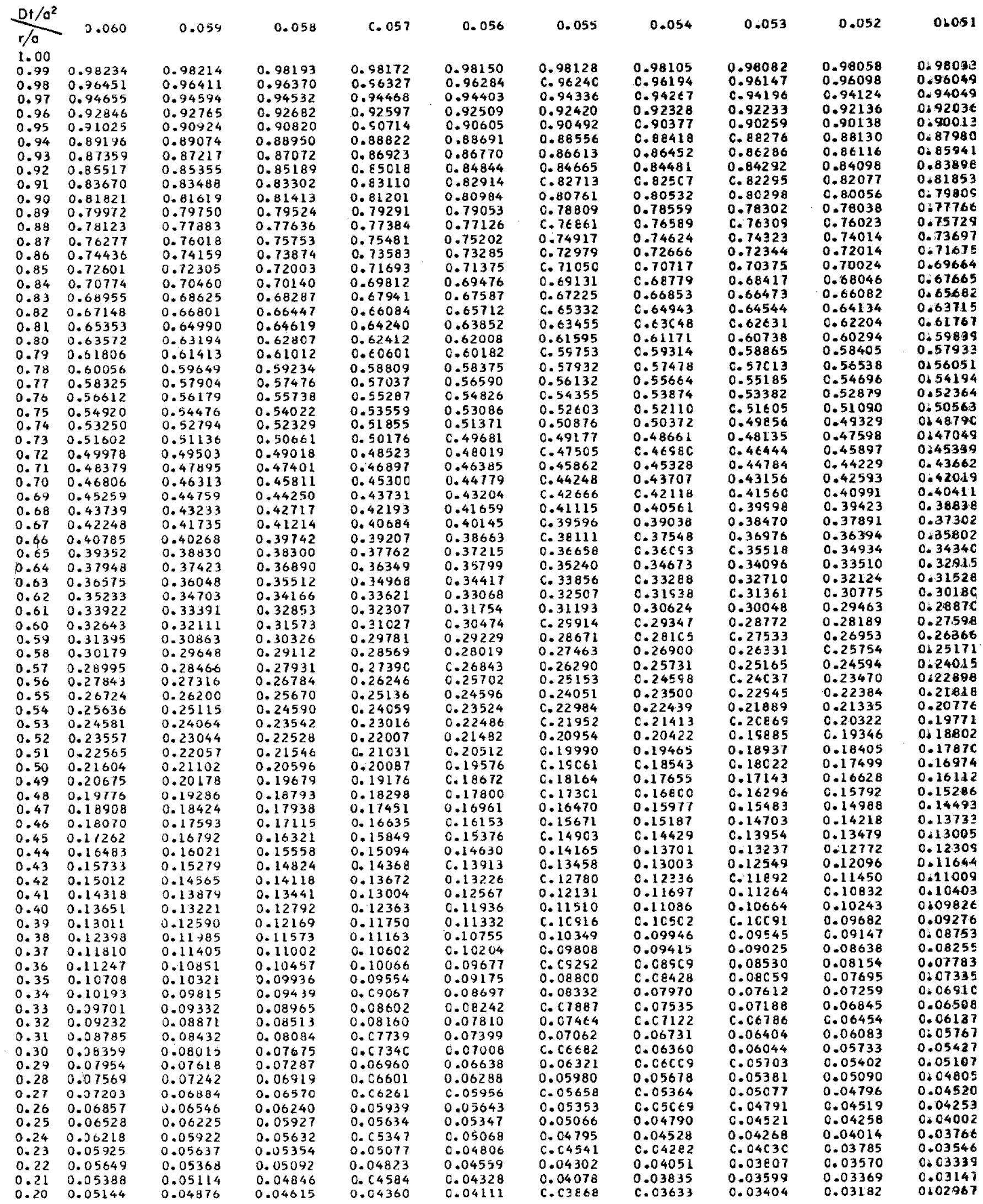


TABLES OF CONCENTRATION RATIOS - $C / c_{0}$

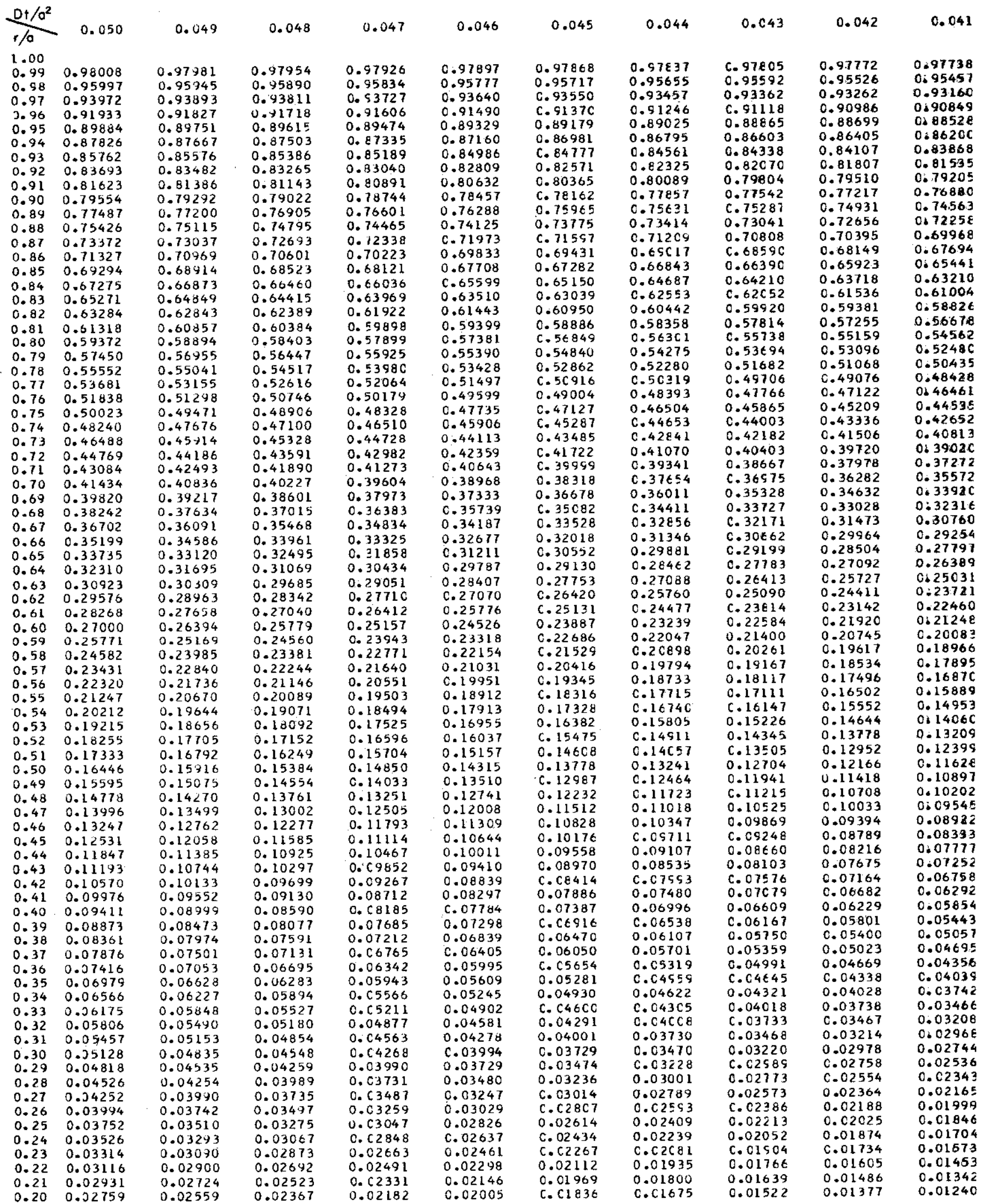


TABLES OF CONCENTRATION RATIOS $-c / c_{0}$

\begin{tabular}{|c|c|c|c|c|c|c|c|c|c|c|}
\hline$\frac{D t / a^{2}}{r / a}$ & 0.040 & 0.039 & 0.038 & 0.037 & 0.036 & 0.035 & C.C34 & 0.033 & 0.032 & 0.031 \\
\hline $\begin{array}{l}1.00 \\
0.99\end{array}$ & 0.97703 & 0.97666 & 0.97628 & 0.97589 & 0.97547 & C. 97505 & $0.974<0$ & C. 97413 & 0.97364 & 0.97313 \\
\hline 0.98 & 0.95386 & 0.95313 & 0.95236 & 0.95157 & 0.95074 & 0.94988 & $\begin{array}{l}0.94898 \\
0.92320\end{array}$ & $\begin{array}{l}0.94805 \\
0.92179\end{array}$ & $\begin{array}{l}0.94706 \\
0.92032\end{array}$ & $\begin{array}{l}0.94604 \\
0.91877\end{array}$ \\
\hline $\begin{array}{l}0.97 \\
0.96\end{array}$ & $\begin{array}{l}0.93053 \\
0.90707\end{array}$ & $\begin{array}{l}0.92943 \\
0.90559\end{array}$ & $\begin{array}{l}3.92828 \\
0.90406\end{array}$ & $\begin{array}{l}0.52708 \\
0.90247\end{array}$ & $\begin{array}{l}0.92584 \\
0.90081\end{array}$ & $\begin{array}{l}0.92455 \\
C .89909\end{array}$ & $\begin{array}{l}0.92320 \\
0.89729\end{array}$ & $\begin{array}{l}0.92179 \\
0.89541\end{array}$ & 0.89344 & 0.89138 \\
\hline 0.95 & 0.88350 & 0.88166 & 0.87975 & 0.87776 & 0.87569 & 0.87353 & 0.87129 & 0.86894 & 0.86649 & 0.86392 \\
\hline 0.94 & 0.85987 & 0.85766 & 0.85537 & $0 . \& 5299$ & 0.85051 & 0.84793 & 0.84524 & 0.84244 & 0.83950 & 0.83643 \\
\hline 0.93 & 0.83620 & 0.83363 & 0.83097 & 0.82820 & 0.82532 & C. 82232 & $0.8192 \mathrm{C}$ & 0.81594 & 0.81253 & 0.80896 \\
\hline 0.92 & 0.81253 & 0.80961 & 0.80657 & 0.80342 & 0.80015 & 0.79674 & 0.79319 & 0.78948 & 0.78561 & 0.78157 \\
\hline 0.91 & 0.78889 & 0.78562 & 0.78223 & 0.77870 & 0.77504 & 0.77123 & 0.76726 & 0.76312 & 0.75880 & 0.75428 \\
\hline 0.90 & 0.70531 & 0.76170 & 0.75796 & 0.75407 & 0.75003 & C. 74583 & C. 74145 & 0.73690 & 0.73214 & 0.72 .717 \\
\hline 0.89 & 0.74183 & 0.73788 & 0.73380 & 0.72956 & 0.72515 & 0.72057 & 0.71581 & C. $71 C 84$ & 0.70567 & 0.70026 \\
\hline 0.88 & 0.71847 & 0.71420 & 0.70978 & 0.70520 & 0.70045 & 0.69550 & 0.69036 & 0.68501 & 0.67943 & $086736 \mathrm{C}$ \\
\hline 0.87 & & 0.69069 & 0.68595 & $0 . \in 8104$ & 0.67595 & $c .67065$ & 0.66515 & 0.65942 & 0.65346 & 0.64723 \\
\hline 0.86 & 0.67223 & 0.66737 & 0.66232 & 0.65710 & 0.65168 & 0.64606 & $0.64 C 21$ & C. 63413 & 0.62780 & 0.62120 \\
\hline 0.85 & 0.64942 & 0.64427 & 0.63894 & 0.63342 & 0.62769 & 0.62175 & 0.61558 & 0.60917 & 0.60250 & \\
\hline $\begin{array}{l}0.84 \\
0.83\end{array}$ & $\begin{array}{l}0.62685 \\
0.60454\end{array}$ & & & 0.61002 & 0.60400 & 0.59777 & & $\begin{array}{l}0.58457 \\
C .56 C 36\end{array}$ & $\begin{array}{l}0.57758 \\
0.55308\end{array}$ & $\begin{array}{l}0.57030 \\
0.54550\end{array}$ \\
\hline $\begin{array}{l}0.83 \\
0.82\end{array}$ & $\begin{array}{l}0.00434 \\
0.58253\end{array}$ & 0.57662 & $\begin{array}{l}0.59300 \\
0.57050\end{array}$ & $\begin{array}{l}0.58691 \\
0.56419\end{array}$ & $\begin{array}{l}0.58065 \\
0.55765\end{array}$ & $\begin{array}{l}0.57413 \\
0.55088\end{array}$ & $\begin{array}{l}0.56738 \\
0.54386\end{array}$ & $\begin{array}{l}0.56 C 36 \\
0.53658\end{array}$ & 0.52903 & 0.521 .17 \\
\hline 0.81 & 0.56083 & 0.55469 & 0.54836 & 0.54181 & 0.53504 & 0.52803 & 0.52078 & 0.51326 & 0.50546 & 0.49736 \\
\hline 0.80 & 0.53947 & 0.53313 & 0.52658 & 0.51982 & 0.51284 & c. 5C562 & 0.49815 & C. 49641 & 0.48239 & 0.47407 \\
\hline 0.79 & 0.51847 & 0.51194 & 0.50520 & 0.49825 & 0.49108 & 0.48366 & 0.47600 & 0.46807 & 0.45986 & 0845135 \\
\hline 0.78 & 0.49784 & 0.49114 & 0.48424 & 0.47712 & C. 46977 & C. 46219 & 0.45435 & 0.44626 & 0.43788 & 0.42921 \\
\hline 0.77 & 0.47762 & 0.47077 & 0.46371 & 0.45644 & C. 44894 & C.44121 & C. 43323 & 0.42499 & 0.41647 & 0.40767 \\
\hline 0.76 & 0.45781 & 0.45082 & 0.44363 & 0.43623 & 0.42860 & 0.42075 & 0.41264 & 0.40429 & 0.39566 & 0.38675 \\
\hline 0.75 & 0.43844 & 0.43133 & 0.42402 & 0.41651 & 0.40878 & C. 40081 & 0.39261 & 0.38416 & 0.37545 & $0.36,64 t$ \\
\hline 0.74 & 0.41950 & 0.41230 & 0.40490 & 0.39729 & 0.38947 & C. 38143 & c.37315 & 0.36464 & 0.35586 & 0.34683 \\
\hline 0.73 & 0.40103 & 0.39374 & 0.38627 & 0.37859 & 0.37070 & 0.36260 & c.35427 & 0.34571 & 0.33690 & 0.32784 \\
\hline 0.72 & & 0.37568 & 0.36814 & 0.36041 & 0.35248 & C. 34434 & 0.33598 & 0.32740 & 0.31858 & 0.30952 \\
\hline 0.71 & 0.36550 & 0.35811 & 0.35053 & 0.34277 & 0.33481 & C. 32665 & C. 31829 & 0.30971 & 0.30091 & 0.29187 \\
\hline 0.70 & 0.34846 & 0.34104 & 0.33344 & 0.32566 & 0.31770 & 0.30955 & $0.3 \mathrm{C} 120$ & C.292.64 & 0.28388 & 0.27489 \\
\hline 0.69 & 0.33192 & 0.32448 & 0.31688 & 0.30911 & 0.30116 & 0.29303 & 0.28471 & 0.27620 & 0.26749 & 0.25859 \\
\hline 0.68 & 0.31588 & 0.30844 & 0.30085 & $0.2931 \mathrm{C}$ & 0.28518 & C. 27709 & 0.26883 & c.26039 & 0.25176 & $0.2429 E$ \\
\hline 0.67 & 0.30034 & 0.29292 & 0.28536 & 0.27765 & 0.26978 & 0.26175 & 0.25355 & C. $2452 \mathrm{C}$ & 0.23667 & 0.22797 \\
\hline 0.66 & 0.28530 & 0.27792 & 0.27041 & 0.26275 & 0.25494 & 0.24699 & 0.23888 & 0.23063 & 0.22222 & 0.21368 \\
\hline 0.65 & 0.27077 & 0.26344 & 0.25599 & 0.24840 & C.24067 & C. 23281 & C.22481 & 0.21668 & 0.20841 & 0.20000 \\
\hline 0.64 & 0.25675 & 0.24949 & 0.24210 & 0.23460 & 0.22697 & 0.21921 & 0.21134 & C. 20334 & 0.19522 & 0,18698 \\
\hline 0.63 & 0.24323 & 0.23604 & 0.22875 & 0.22134 & 0.21382 & 0.20619 & 0.19845 & 0.19060 & 0.18265 & $0.1745 \mathrm{~s}$ \\
\hline 0.62 & 0.23022 & 0.22312 & 0.21592 & 0.20862 & 0.20123 & 0.19373 & 0.18614 & 0.17846 & 0.17088 & 0.16282 \\
\hline 0.61 & 0.21770 & 0.21070 & 0.20362 & 0.19644 & 0.18918 & C.18183 & 0.17440 & C. 16689 & 0.15931 & 0.15165 \\
\hline 0.60 & 0.20568 & 0.19879 & 0.19183 & 0.18479 & 0.17767 & 0.17048 & 0.16322 & 0.15590 & 0.14852 & 0.14108 \\
\hline 0.59 & 0.19414 & 0.18738 & 0.18055 & 0.17365 & 0.16669 & 0.15967 & 0.15259 & 0.14546 & 0.13829 & 0.13108 \\
\hline 0.58 & 0.18309 & 0.17646 & 0.16977 & 0.16302 & 0.15622 & C. 14538 & 0.14249 & 0.13557 & 0.12862 & 0.12164 \\
\hline 0.57 & 0.17251 & 0.16602 & 0.15948 & 0.15289 & 0.14627 & 0.13961 & 0.13292 & 0.12620 & 0.11948 & 0.11274 \\
\hline 0.56 & 0.16239 & 0.15605 & 0.14966 & 0.14325 & C. 13680 & 0.13033 & 0.12385 & 0.11735 & 0.11085 & $0.1043 t$ \\
\hline 0.55 & 0.15273 & 0.14654 & 0.14032 & 0.13408 & c. 12782 & C. 12155 & 0.11527 & 0.10899 & 0.10273 & 0.09648 \\
\hline 0.54 & 0.14352 & 0.13749 & 0.13144 & 0.12537 & 0.11930 & 0.11323 & 0.10717 & c. 10112 & 0.09509 & 0.08909 \\
\hline 0.53 & 0.13474 & 0.12888 & 0.12300 & 0.11712 & 0.11124 & 0.10537 & 0.09952 & 0.09370 & 0.08791 & 0.08217 \\
\hline 0.52 & 0.12639 & 0.12069 & 0.11499 & $0.1093 \mathrm{C}$ & 0.10362 & C. $c 5796$ & $C .09233$ & 0.08673 & 0.08118 & 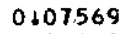 \\
\hline 0.51 & 0.11845 & 0.11292 & 0.10740 & 0.10190 & 0.04642 & 0.09097 & C. C8556 & C. $0.8 C 19$ & 0.07488 & 0.06963 \\
\hline 0.50 & 0.11092 & 0.10556 & 0.10022 & 0.09491 & 0.08963 & 0.08439 & 0.07920 & 0.07406 & 0.06898 & 0.06398 \\
\hline 0.49 & 0.10377 & 0.09859 & 0.09344 & 0.68832 & 0.08324 & 0.07821 & C.c7323 & 0.06832 & 0.06348 & 0.05872 \\
\hline 0.48 & 0.09700 & 0.09200 & 0.08703 & 0.08210 & 0.07722 & 0.07240 & $0.067 \in 4$ & C. 06295 & 0.05834 & 0.05382 \\
\hline 0.47 & 0.09059 & 0.08577 & 0.08099 & 0.07625 & 0.07157 & 0.06696 & 0.06241 & 0.05794 & 0.05356 & $0.0492 \varepsilon$ \\
\hline 0.46 & 0.08453 & 0.07989 & 0.07529 & 0.07075 & 0.06627. & C. 06186 & 0.05752 & 0.05327 & 0.04912 & 0.04506 \\
\hline 0.45 & 0.07882 & 0.07435 & 0.06994 & 0.06559 & 0.06130 & C.C5709 & 0.05256 & C. 04893 & 0.04499 & 0.04116 \\
\hline 0.44 & 0.57343 & 0.06914 & 0.06491 & c. $C 6 C 74$ & c. 05665 & c. 05264 & 0.04872 & 0.04489 & 0.04116 & 0.03755 \\
\hline 0.43 & 0.06835 & 0.06423 & 0.06018 & 0.05620 & 0.05230 & 0.04849 & c.C4476 & C. 04114 & 0.03762 & 0.03421 \\
\hline 0.42 & 0.06357 & 0.05963 & 0.05575 & 0.05196 & 0.04824 & 0.04462 & 0.04109 & $0.0376 t$ & C.03434 & 0.03114 \\
\hline 0.41 & 0.05908 & 0.05531 & 0.05161 & 0.04795 & 0.04446 & 0.04102 & 0.03767 & 0.03444 & 0.03131 & 0.02831 \\
\hline 0.40 & 0.05486 & 0.05126 & 0.04773 & 0.04429 & 0.04093 & c. $c 3767$ & 0.03451 & c. 03146 & c. 02852 & 0.02571 \\
\hline 0.39 & 0.05091 & 0.04747 & 0.04411 & 0.04083 & 0.03765 & 0.03456 & 0.03158 & 0.02871 & 0.02595 & 0.02332 \\
\hline 0.38 & 0.04720 & 0.04392 & 0.04073 & c. 03762 & 0.0346 & 0.03169 & 0.02888 & 0.02618 & 0.02359 & 0.02113 \\
\hline 0.37 & 0.54374 & 0.04061 & 0.03757 & 0.03463 & 0.03177 & C. $c 2902$ & 0.02638 & 0.02384 & 0.02142 & 0.01912 \\
\hline 0.36 & 0.04050 & 0.03753 & 0.03464 & 0.03185 & 0.02915 & 0.026 & $C . C 24 C 7$ & 0.02169 & 0.01943 & 0.01729 \\
\hline 0.35 & 0.03747 & 0.03465 & 0.03191 & 0.02927 & 0.02672 & 0.024 & 0.02195 & 0.01972 & 0.01761 & $0: 01562$ \\
\hline 0.34 & 0.03465 & 0.03197 & 0.02938 & 0.02688 & 0.02448 & C. C2218 & c.c1999 & 0.01791 & 0.01594 & 0.01409 \\
\hline 0.33 & 0.03202 & 0.02948 & 0.02702 & 0.02467 & 0.02241 & $c .02025$ & C.c122c & $c .01625$ & 0.01442 & $0.0127 \mathrm{C}$ \\
\hline 0.32 & 0.02958 & 0.02716 & 0.02484 & 0.02262 & 0.02049 & 0.01847 & 0.01655 & 0.01473 & 0.01303 & 0.01144 \\
\hline 0.31 & 0.02730 & 0.02502 & 0.02283 & 0.02073 & 0.01873 & C. $C 1 \in 83$ & $0.015 C_{4}$ & 0.01335 & 0.01176 & 0.01029 \\
\hline 0.30 & 0.02519 & 0.02303 & 0.02096 & 0.01899 & 0.01711 & 0.01533 & C.c13t5 & C. 01208 & 0.01061 & 0.00925 \\
\hline 0.29 & 0.02323 & 0.02119 & 0.01924 & 0.01738 & 0.01562 & 0.01395 & 0.01239 & $0.01<93$ & 0.00957 & 0.00831 \\
\hline 0.28 & 0.32141 & 0.01748 & 0.01765 & C.C159C & 0.01425 & 0.01269 & C.C1124 & 0.00988 & 0.00862 & 0.00745 \\
\hline 0.27 & 0.01973 & 0.01791 & 0.01618 & 0.01454 & 0.01299 & 0.01154 & c.clclo & C. OCE 92 & 0.00775 & 0.00668 \\
\hline 0.26 & 0.01818 & 0.01646 & 0.01483 & 0.01329 & 0.01184 & 0.01049 & 0.00922 & 0.00805 & 0.00698 & 0.00595 \\
\hline 0.25 & 0.01675 & 0.01512 & 0.01359 & $0 . c 1215$ & 0.01079 & C. 00953 & 0.00835 & 0.00727 & 0.00627 & $0.0053 t$ \\
\hline 0.24 & 0.01542 & 0.01389 & 0.01245 & 0.01110 & 0.00983 & C. CC 865 & C.co7s 5 & c. $c 0 \in 55$ & 0.00564 & 0.00480 \\
\hline 0.23 & 0.01420 & 0.01276 & 0.01141 & 0.01014 & 0.00895 & 0.00785 & 0.00684 & 0.00591 & 0.00506 & $0: 00429$ \\
\hline 0.22 & 0.01308 & 0.01172 & 0.01045 & o. $\cos 26$ & C. 00815 & $c .00713$ & 0.00619 & 0.00533 & 0.00455 & 0.00384 \\
\hline 0.21 & 0.01205 & 0.01077 & 0.00958 & 0.00846 & 0.00743 & C. $C[\in \in 47$ & c.ccs 60 & C. $0048 \mathrm{C}$ & 0.00408 & 0.00343 \\
\hline 0.20 & 0.01111 & 0.00490 & 0.00878 & 0.00773 & 0.00676 & 0.00588 & 0.00506 & c.000433 & 0.00366 & 0,00307 \\
\hline
\end{tabular}


TABLES OF CONCENTRATION RATIOS - $c / c_{0}$

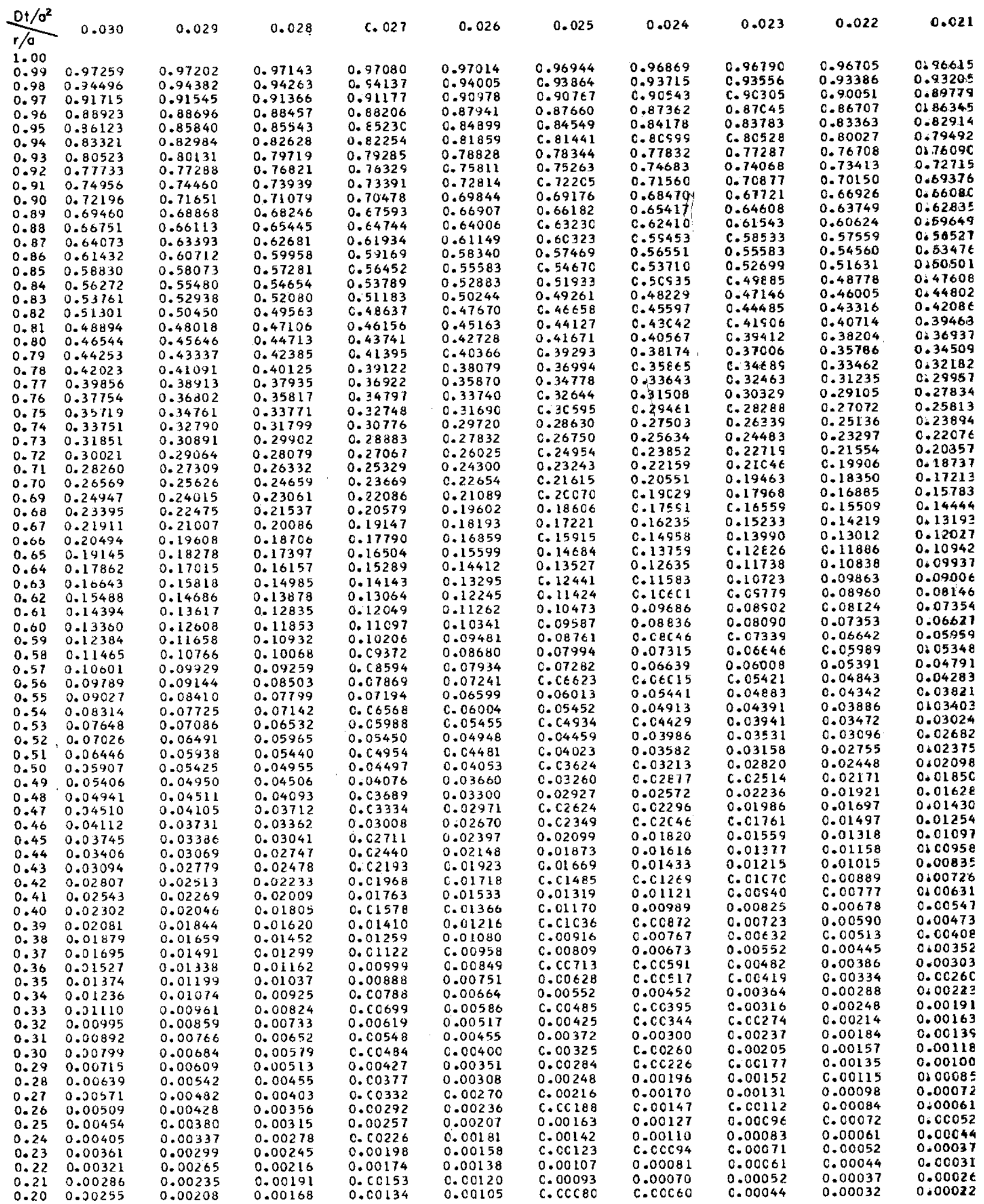


TABLES OF CONCENTRATION RATIOS - $c / c_{0}$

\begin{tabular}{|c|c|c|c|c|c|c|c|c|c|c|}
\hline$\frac{D t / 0^{2}}{r / 0}$ & 0.020 & 0.019 & 0.018 & 0.017 & 0.016 & 0.015 & C.C14 & 0.013 & 0.012 & 0.011 \\
\hline $\begin{array}{l}1.00 \\
0.99\end{array}$ & & & & & & & & & & \\
\hline 0.98 & $\begin{array}{l}0.96518 \\
0.93010\end{array}$ & $\begin{array}{l}0.96413 \\
0.92801\end{array}$ & $\begin{array}{l}0.96300 \\
0.92574\end{array}$ & $\begin{array}{l}0.96177 \\
0.92328\end{array}$ & $\begin{array}{l}0.96042 \\
0.92059\end{array}$ & $\begin{array}{l}\text { C. } 55895 \\
0.91764\end{array}$ & $\begin{array}{l}0.95732 \\
0.91438\end{array}$ & $\begin{array}{r}\text { C. } 95551 \\
0.91075\end{array}$ & $\begin{array}{l}0.95347 \\
0.90609\end{array}$ & $\begin{array}{l}0.95117 \\
0190208\end{array}$ \\
\hline 0.97 & 0.89487 & 0.89173 & 0.88833 & 0.88465 & 0.88062 & 0.87621 & 0.87134 & 0.86592 & 0.85986 & 0.85295 \\
\hline 0.96 & 0.85958 & 0.85541 & 0.85090 & 0.84601 & 0.84067 & C. 83482 & 0.82837 & $0.8212 \mathrm{I}$ & 0.81319 & 0280413 \\
\hline 0.95 & 0.82432 & 0.81914 & 0.81354 & 0.80747 & 0.80086 & 0.79361 & 0.78563 & 0.77678 & 0.76688 & 0.75572 \\
\hline 0.94 & 0.78919 & 0.78302 & 0.77637 & 0.76916 & 0.76132 & 0.75273 & 0.74329 & 0.73282 & 0.72114 & 0.707 .99 \\
\hline 0.93 & 0.75428 & 0.74717 & 0.73950 & 0.73120 & 0.72218 & C. 11232 & 0.70149 & 0.68950 & 0.67615 & 0.661116 \\
\hline 0.92 & 0.71968 & 0.71167 & 0.70303 & 0.69370 & 0.68357 & 0.67251 & 0.66037 & C. 64698 & 0.63210 & 0.61542 \\
\hline 0.91 & 0.68548 & 0.67661 & 0.66707 & 0.65676 & 0.64559 & 0.63342 & 0.62009 & 0.60541 & 0.58914 & $0: 57096$ \\
\hline 0.90 & 0.65177 & 0.64209 & 0.63170 & 0.62050 & 0.60837 & C. 55518 & 0.58077 & 0.56494 & 0.54743 & 0.5279 .5 \\
\hline 0.89 & 0.61861 & 0.60820 & 0.59702 & 0.58499 & 0.57200 & 0.55789 & 0.54252 & 0.52568 & 0.50711 & 0.48652 \\
\hline 0.88 & 0.58610 & 0.57500 & 0.56312 & 0.55035 & 0.53658 & 0.52167 & 0.50546 & 0.48775 & 0.46830 & 02447681 \\
\hline 0.87 & 0.55429 & 0.54258 & 0.53006 & 0.51663 & 0.50219 & 0.48659 & C. 46967 & 0.45125 & 0.43109 & 0640891 \\
\hline 0.86 & 0.52325 & 0.51100 & 0.49792 & 0.48393 & 0.46891 & 0.45273 & 0.43524 & $C .4162 \theta$ & 0.39557 & 0837292 \\
\hline 0.85 & 0.49304 & 0.48032 & 0.46677 & 0.45230 & 0.43681 & 0.42017 & 0.40224 & 0.38285 & 0.36182 & $0.3389 \mathrm{C}$ \\
\hline 0.84 & 0.46371 & 0.45059 & 0.43665 & 0.42180 & 0.40594 & C. 38896 & 0.37072 & 0.35108 & 0.329 .86 & $0 \$ 30688$ \\
\hline 0.83 & 0.43532 & 0.42187 & 0.40762 & 0.39248 & 0.37635 & 0.35914 & 0.34072 & C. $32 \mathrm{C98}$ & 0.29975 & 0227688 \\
\hline 0.82 & 0.40789 & 0.39420 & 0.37971 & 0.36437 & 0.34808 & 0.33075 & 0.31228 & 0.29256 & 0.27148 & 0.24891 \\
\hline 0.81 & 0.38146 & 0.36760 & 0.35296 & 0.33751 & 0.32115 & C. 30381 & 0.28541 & 0.26585 & 0.24506 & 0.22294 \\
\hline 0.80 & 0.35607 & 0.34210 & 0.32739 & 0.31191 & C.29557 & C. 27833 & 0.26010 & $C .24 C 84$ & 0.22047 & 0.19894 \\
\hline 0.79 & 0.33173 & 0.31772 & 0.30302 & 0.28759 & 0.27137 & 0.25431 & 0.23637 & 0.21750 & 0.19767 & 0.17681 \\
\hline 0.78 & 0.30845 & 2.29447 & 0.27985 & 0.26455 & C. 24852 & C. 23175 & 0.21418 & 0.19581 & 0.17663 & 0.15665 \\
\hline 0.77 & 0.28625 & 0.27236 & 0.25788 & 0.24278 & 0.22704 & 0.21062 & 0.19352 & 0.17573 & 0.1572 & 0.13823 \\
\hline 0.76 & 0.26512 & 0.25139 & 0.23712 & 0.22229 & 0.20689 & 0.19090 & 0.17434 & 0.15721 & 0.13958 & $0.1215 \mathrm{C}$ \\
\hline 0.75 & 0.24507 & 0.23155 & 0.21755 & 0.20304 & C. 18805 & 0.17256 & 0.15660 & 0.14020 & 0.12343 & $0.1064 \mathrm{C}$ \\
\hline 0.74 & 0.22609 & 0.21283 & 0.19914 & 0.18503 & 0.17049 & C. 15555 & $0.14 C 25$ & 0.12463 & 0.10878 & 0.09281 \\
\hline 0.73 & 0.20817 & 0.19521 & 0.18189 & 0.16820 & 0.15418 & 0.13984 & 0.12524 & C.11C43 & 0.09552 & 0208065 \\
\hline 0.72 & 0.19128 & 0.17867 & 0.16575 & 0.15255 & 0.13907 & 0.12537 & 0.11150 & 0.09754 & 0.08359 & 0.06981 \\
\hline & 0.17541 & 0.16319 & 0.15071 & 0.13801 & 0.12512 & c. 11208 & 0.09897 & 0.08587 & 0.07289 & 0.06049 \\
\hline 0.70 & 0.16054 & 0.14873 & 0.13672 & 0.12456 & 0.11228 & 0.09993 & 0.08759 & C.07535 & 0.06333 & 0.0 .5169 \\
\hline 0.69 & 0.14662 & 0.13526 & 0.12375 & 0.11215 & 0.10049 & 0.08884 & 0.07728 & 0.06590 & 0.05483 & 0104422 \\
\hline 0.68 & 0.13365 & 0.12274 & 0.11175 & 0.10073 & 0.08971 & c. 07877 & 0.06798 & 0.05745 & 0.04730 & 0403768 \\
\hline 0.67 & 0.12157 & 0.11114 & 0.10069 & 0.09025 & 0.07987 & 0.06964 & 0.05962 & c. 04992 & 0.04066 & 0.03198 \\
\hline 0.66 & 0.11036 & 0.10043 & 0.09051 & $0 . C 8066$ & 0.07093 & 0.06139 & 0.05213 & 0.04323 & 0.03483 & 0.02704 \\
\hline 0.65 & 0.09997 & 0.09054 & 0.08117 & 0.67192 & 0.06282 & C. 05397 & 0.04544 & 0.03732 & 0.02972 & 0.0227 .7 \\
\hline 0.64 & 0.09038 & 0.08146 & 0.07263 & 0.06396 & 0.05550 & $c . c 4731$ & 0.03548 & $0.0321 \mathrm{C}$ & 0.02527 & 0.01909 \\
\hline 0.63 & 0.08154 & 0.07312 & 0.06484 & 0.05674 & 0.04889 & 0.04136 & 0.03421 & 0.02753 & 0.02141 & 0.01595 \\
\hline 0.62 & 0.07342 & 0.06550 & 0.05775 & $0 . C 5022$ & 0.04296 & 0.03605 & 0.02954 & 0.02352 & 0.01807 & 0.01327 \\
\hline 0.61 & 0.06596 & 0.05854 & 0.05131 & 0.04433 & 0.03765 & C.C3133 & 0.02544 & 0.02004 & 0.01520 & 0.01100 \\
\hline 0.60 & 0.05915 & 0.05221 & 0.04549 & 0.03904 & 0.032 & 0.02715 & 0.021 & 0.01 & 0.01274 & \\
\hline 0.59 & 0.05 & 0.04646 & 0.04023 & 0.03429 & 0.02869 & c. 02347 & 0.01868 & 0.01439 & 0.01063 & $0.0074 t$ \\
\hline 0.58 & 0.04726 & 0.04125 & 0.03550 & 0.03005 & 0.02494 & C. 02022 & c. 01594 & 0.01213 & 0.00885 & 0.00611 \\
\hline 0.57 & 0.04211 & 0.03654 & 0.03125 & 0.02626 & 0.02162 & 0.01737 & $c .01355$ & $c .01620$ & 0.00733 & 0.00498 \\
\hline 0.56 & 0.03744 & 0.03230 & 0.027 & 02290 & 0.01870 & 0.01489 & 0.01149 & 0.00854 & 0.00605 & 0.00404 \\
\hline 0.55 & 0.03323 & 0.02849 & 0.02405 & 0.01991 & 0.01613 & C. C1272 & $0 . \cos 71$ & 0.00713 & 0.00498 & 0.00327 \\
\hline 0.54 & 0.02942 & 0.02508 & 0.02102 & 0.01727 & 0.01387 & 0.01083 & 0.00818 & $C .0 C 593$ & 0.004 & 0.00263 \\
\hline 0.53 & 0.02600 & 0.02202 & 0.01833 & 0.01495 & 0.01190 & 0.00920 & 0.00687 & 0.00492 & 0.00333 & 0600211 \\
\hline 0.52 & 0.32293 & 0.01930 & 0.01595 & 0.01290 & 0.01018 & C. $c 0779$ & 0.00575 & 0.00406 & 0.00271 & $0 \$ 00169$ \\
\hline 0.51 & 0.02018 & 0.01687 & 0.01384 & 0.01111 & 0.00869 & 0.00658 & 0.00480 & C. 00335 & 0.00220 & 0.00134 \\
\hline 0.50 & 0.01772 & 0.01472 & 0.01199 & 0.00954 & 0.00739 & 0.00554 & 0.00400 & 0.00275 & 0.00178 & $0: 0010 t$ \\
\hline 0.49 & 0.01553 & 0.01281 & 0.01036 & 0.00817 & 0.00027 & c. 00465 & 0.00331 & 0.00225 & 0.00143 & 0.00084 \\
\hline 0.48 & 0.01358 & 0.01113 & 0.00893 & 0.00699 & 0.00531 & 0.00390 & c.cc2 274 & C. 00183 & 0.00115 & 0.00066 \\
\hline 0.47 & 0.01186 & 0.00464 & 0.00768 & 0.00596 & 0.00448 & 0.00325 & 0.00226 & 0.00149 & 0.00092 & 0100052 \\
\hline 0.46 & 0.01033 & 0.00834 & 0.00659 & c. 00506 & c. 00377 & C. 00271 & 0.00186 & 0.00120 & 0.00073 & 0.00040 \\
\hline 0.45 & 0.00898 & 0.00720 & 0.00564 & 0.00430 & 0.00317 & C. $\cos 225$ & c.cc152 & C. 00697 & 0.00058 & 0.00031 \\
\hline 0.44 & 0.00779 & 0.00620 & 0.00481 & 0.003 & 0.00265 & 0.001 & & & & $0: 00$ \\
\hline 0.43 & 0.00674 & 0.00532 & 0.00410 & 0.00307 & 0.00222 & c. 00154 & 0.00101 & 0.00063 & 0.00036 & 0200019 \\
\hline 0.42 & 0.00582 & 0.00456 & 0.00349 & 0.00258 & 0.00185 & 0.00126 & $0 . \operatorname{ccc} \varepsilon 2$ & $c \cdot \operatorname{coc} 5 c$ & 0.00028 & 0.00014 \\
\hline 0.41 & 0.00502 & 0.00390 & 0.00296 & 0.00217 & 0.0015 & 0.001 & 0.000 & 0.00 & 0.00 & 0.00011 \\
\hline 0.40 & 0.00432 & 0.00333 & 0.00250 & 0.00182 & 0.00127 & c. 00085 & 0.00054 & 0.00032 & 0.00017 & 0.00008 \\
\hline 0.39 & 0.00371 & 0.00284 & 0.00211 & 0.00152 & 0.00105 & 0.00069 & 0.00043 & c. $\operatorname{ccc} 25$ & 0.00013 & 0.00006 \\
\hline 0.38 & 0.00318 & 0.00241 & 0.00178 & $0 . \operatorname{col} 27$ & 0.000 & 0.000 & 0.000 & 0.00 & 0.00 & 0.00005 \\
\hline 0.37 & 0.00272 & 0.00205 & 0.00150 & 0.00105 & c. 00071 & 0.000 & 0.000 & 0.000 & 0.00 & 0.00 \\
\hline 0.36 & 0.00232 & 0.00173 & 0.00125 & 0.00087 & 0.00058 & C. $\operatorname{ccc37}$ & $0 . \operatorname{ccc} 22$ & c. coc12 & 0.00006 & 0.00003 \\
\hline 0.35 & 0.06198 & 0.00146 & 0.00105 & 0.00072 & 0.000 & 0.000 & 0.00018 & 0.00009 & 0.000 & 0.00002 \\
\hline 0.34 & 0.00168 & 0.00123 & 0.00088 & 0.00060 & c. 00039 & 0.000 & 0.000 & 0.000 & 0.000 & 0.00002 \\
\hline 0.33 & 0.00143 & 0.00104 & 0.00073 & 0.00049 & 0.00032 & C. Ccclo & $0 . \operatorname{cocll}$ & 0.00006 & 0.00003 & $0 \$ 00001$ \\
\hline 0.32 & 0.00121 & 0.00087 & 0.00061 & 0.00041 & 0.00026 & 0.00015 & $0 . \operatorname{cccs}$ & 0.00004 & 0.00002 & 0.00001 \\
\hline 0.31 & 0.00102 & 0.00073 & 0.00050 & 0.00033 & 0.00021 & 0.00012 & 0.000 & 0.000 & 0.000 & 0.00001 \\
\hline 0.30 & 0.30086 & 0.00061 & 0.000 & 0.00027 & $c .00017$ & C. $c 0010$ & c. 00005 & 0.00003 & 0.00001 & 0,00000 \\
\hline 0.29 & 0.00073 & 0.00051 & 0.00034 & 0.00022 & 0.00014 & 0.00008 & $\mathrm{C.cccc} 4$ & c. CcCO2 & 0.00001 & $0.00 \mathrm{coc}$ \\
\hline 0.28 & 0.00061 & 0.00043 & 0.00028 & 0.00018 & 0.00011 & 0.00006 & 0.00003 & & 0.00001 & $0 \$ 0000 C$ \\
\hline 0.27 & 0.30051 & 0.00035 & 0.00023 & 0.00015 & 0.00009 & C. 00005 & c. $\operatorname{cccc} 3$ & 0.00001 & 0.00001 & 0200000 \\
\hline 0.26 & 0.00043 & 0.00029 & 0.00019 & 0.00012 & 0.00007 & 0.00004 & $0 . \operatorname{ccc} 2$ & c.cccol & 0.00000 & 0.00000 \\
\hline 0.25 & 0.00036 & 0,00024 & 0.00016 & 0.00010 & 0.00006 & 0.00003 & 0.00002 & 0.00001 & 0.00000 & $0.0000 \mathrm{C}$ \\
\hline 0.24 & 0.00030 & 0.00020 & 0.00013 & 0.00008 & c.00005 & C. 00002 & C.0OCCl & 0.00001 & 0.00000 & 0.00000 \\
\hline 0.23 & 0.00025 & 0.00017 & 0.00011 & 0.00006 & 0.00004 & 0.00002 & C. Cccel & C. COCOC & 0.00000 & $0 \$ 00000$ \\
\hline 0.22 & 0.00021 & 0.00014 & 0.00009 & 0.00005 & 0.00003 & 0.00001 & 0.00001 & 0.00000 & 0.00000 & $0.0000 C$ \\
\hline 0.21 & 0.00018 & 0.00011 & 0.00007 & 0.60004 & 0.00002 & C. 00001 & 0.00001 & 0.00000 & 0.00000 & 0.00000 \\
\hline 0.20 & 0.00015 & 0.00009 & 0.00006 & 0.00003 & 0.00002 & C. CCOOL & c.cccco & C. $00 C 0 \mathrm{C}$ & 0.00000 & 0.00000 \\
\hline
\end{tabular}


TABLES OF CONCENTRATION RATIOS $-\mathrm{c} / \mathrm{c}_{\boldsymbol{a}}$

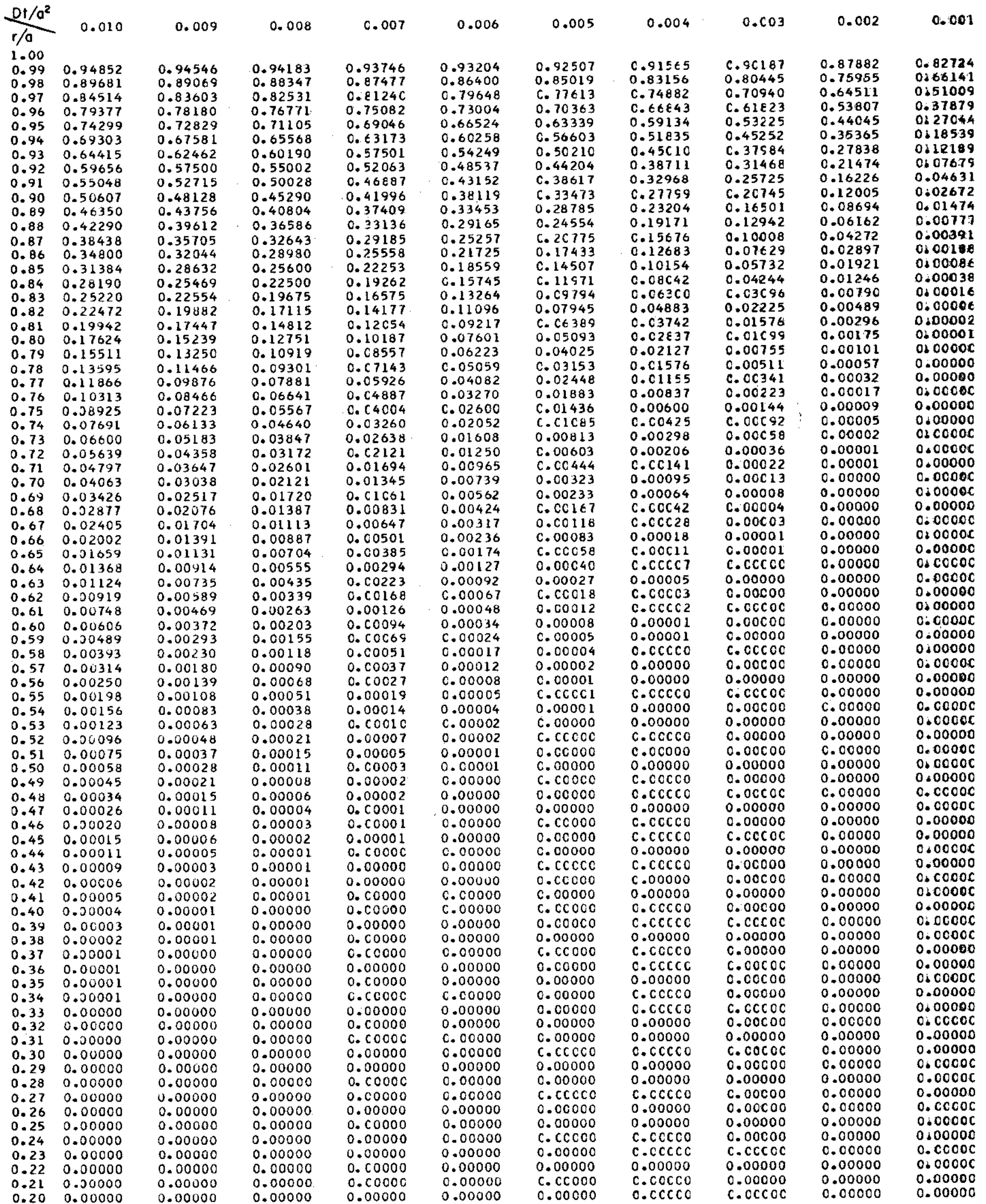

